\title{
MSSM interpretations of the LHC discovery: light or heavy Higgs?
}

\author{
P. Bechtle ${ }^{1, \mathrm{a}}$, S. Heinemeyer ${ }^{2, \mathrm{~b}}$, O. Stål ${ }^{3, \mathrm{c}}$, T. Stefaniak ${ }^{1, \mathrm{~d}}$, G. Weiglein ${ }^{4, \mathrm{e}}$, L. Zeune Zef $^{4, \mathrm{f}}$ \\ ${ }^{1}$ Physikalisches Institut der Universität Bonn, Nußallee 12, 53115 Bonn, Germany \\ ${ }^{2}$ Instituto de Física de Cantabria (CSIC-UC), Santander, Spain \\ ${ }^{3}$ The Oskar Klein Centre, Department of Physics, Stockholm University, 10691 Stockholm, Sweden \\ ${ }^{4}$ Deutsches Elektronen-Synchrotron DESY, Notkestraße 85, 22607 Hamburg, Germany
}

Received: 9 November 2012 / Revised: 23 February 2013 / Published online: 12 April 2013

(c) Springer-Verlag Berlin Heidelberg and Società Italiana di Fisica 2013

\begin{abstract}
A Higgs-like particle with a mass of about $126 \mathrm{GeV}$ has been discovered at the LHC. Within the experimental uncertainties, the measured properties of this new state are compatible with those of the Higgs boson in the Standard Model (SM). While not statistically significant at present, the results show some interesting patterns of deviations from the SM predictions, in particular a higher rate in the $\gamma \gamma$ decay mode observed by ATLAS and CMS, and a somewhat smaller rate in the $\tau^{+} \tau^{-}$mode. The LHC discovery is also compatible with the predictions of the Higgs sector of the Minimal Supersymmetric Standard Model (MSSM), interpreting the new state as either the light or the heavy $\mathcal{C} \mathcal{P}$-even MSSM Higgs boson. Within the framework of the MSSM with seven free parameters (pMSSM-7), we fit the various rates of cross section times branching ratio as measured by the LHC and Tevatron experiments under the hypotheses of either the light or the heavy $\mathcal{C P}$-even Higgs boson being the new state around $126 \mathrm{GeV}$, with and without the inclusion of further low-energy observables. We find an overall good quality of the fits, with the best fit points exhibiting an enhancement of the $\gamma \gamma$ rate, as well as a small suppression of the $b \bar{b}$ and $\tau^{+} \tau^{-}$channels with respect to their SM expectations, depending on the details of the fit. For the fits including the whole dataset the light $\mathcal{C P}$-even Higgs interpretation in the MSSM results in a higher relative fit probability than the SM fit. On the other hand, we find that the present data also permit the more exotic interpretation in terms of the heavy $\mathcal{C P}$-even MSSM
\end{abstract}

\footnotetext{
a e-mail: bechtle@physik.uni-bonn.de

b e-mail: Sven.Heinemeyer@cern.ch

c e-mail: oscar.stal@fysik.su.se

de-mail: tim@th.physik.uni-bonn.de

e e-mail: Georg.Weiglein@ desy.de

f e-mail: lisa.zeune@desy.de
}

Higgs, which could give rise to experimental signatures of additional Higgs states in the near future.

\section{Introduction}

One of the most important goals of the Large Hadron Collider (LHC) is to explore the origin of electroweak symmetry breaking (EWSB). The spectacular discovery of a Higgslike particle with a mass around $\hat{M}_{H} \simeq 126 \mathrm{GeV}$, which has been announced by ATLAS [1] and CMS [2], marks a milestone of an effort that has been ongoing for almost half a century and opens up a new era of particle physics. Both ATLAS and CMS reported a clear excess in the two photon channel, as well as in the $Z Z^{(*)}$ channel. The discovery is further corroborated, though not with high significance, by the $W W^{(*)}$ channel and by the final Tevatron results [3]. The combined sensitivity in each of the LHC experiments reaches more than $5 \sigma$. The observed rate in the $\gamma \gamma$ channel turns out to be considerably above the expectation for a Standard Model (SM) Higgs both for ATLAS [4] and CMS [5], whereas the $b \bar{b}$ and the $\tau^{+} \tau^{-}$channels appear to be somewhat low in the LHC measurements $[6,7]$. While those possible deviations from the SM prediction are not statistically significant at present, so that within the uncertainties the results are compatible with the SM, if confirmed in the future the observed patterns could be a first indication of a non-SM nature of the new state.

Among the most studied candidates for EWSB in the literature are the Higgs mechanism within the SM and within the Minimal Supersymmetric Standard Model (MSSM). Contrary to the SM, two Higgs doublets are required in the MSSM, resulting in five physical Higgs boson degrees of freedom. Without explicit $\mathcal{C P}$-violation in the soft supersymmetry-breaking terms these are the light and heavy 
$\mathcal{C P}$-even Higgs bosons, $h$ and $H$, the $\mathcal{C P}$-odd Higgs boson, $A$, and the charged Higgs boson, $H^{ \pm}$. The Higgs sector of the MSSM can be specified at lowest order in terms of the $Z$ boson mass, $M_{Z}$, the $\mathcal{C P}$-odd Higgs boson mass, $M_{A}$, and $\tan \beta \equiv v_{2} / v_{1}$, the ratio of the two Higgs vacuum expectation values. Including higher-order corrections, the masses of the $\mathcal{C} \mathcal{P}$-even neutral Higgs bosons and the charged Higgs boson can be predicted in terms of the other MSSM parameters; see [8-15] for the original one-loop calculations and [16-18] for reviews.

It was shown that in particular the interpretation of the new state as the light $\mathcal{C P}$-even Higgs boson of the MSSM is a viable possibility (called the "light Higgs case" in the following). The implications and phenomenology of this scenario has been studied in a series of papers [19-50]. On the other hand, it was also pointed out that the heavy $\mathcal{C} \mathcal{P}$ even Higgs boson can have a mass around $126 \mathrm{GeV}$ [19, 20] (called the "heavy Higgs case") while maintaining (within the uncertainties) a SM-like behaviour. All five MSSM Higgs bosons in this scenario would be rather light, and it would in particular imply the existence of another light Higgs with a mass below $126 \mathrm{GeV}$ and suppressed couplings to $W$ and $Z$ bosons. For a recent discussion of the phenomenology of such a scenario, see also [51].

The question arises whether the MSSM (or another model beyond the SM) can give a prediction of the production cross sections and decay widths of the observed Higgslike state that yields a better description of the data than the one provided by the SM. While at the current level of accuracy no clear deviation from the SM can be claimed, the situation could change once the whole dataset of $\sim 20 \mathrm{fb}^{-1}$ to be collected in 2012 is incorporated in the analyses. The main aim of this paper is to investigate in how much the MSSM can improve the theoretical description of the current experimental data, and potentially which parts of the parameter space of the MSSM are favoured by the current experimental data from the various Higgs search channels. To answer these questions we employ a model-dependent fitting approach, in contrast to many analyses of Higgs coupling deviations that have been performed in a model-independent framework [52-74].

Because of the large number of free parameters, the MSSM Higgs search results at LEP [75], the Tevatron [76, 77] and the LHC [78, 79] have been interpreted in certain benchmark scenarios [80-82] (of which the $m_{h}^{\max }$ scenario has been most widely used), where the MSSM parameters entering via higher-order contributions are set to specific fixed benchmark values. However, in order to investigate the potentially favoured regions in the MSSM parameter space a scan over the relevant SUSY parameters has to be performed. A complete scan over the in principle more than 100 free parameters of the MSSM parameter space is neither technically feasible nor does the available experimen- tal information provide sufficient sensitivity to simultaneously constrain a large number of parameters. One therefore needs to focus on a certain subset of parameters. The most ambitious scans performed up to now were done [83, 84] for the phenomenological MSSM with 19 free parameters (pMSSM-19, see [85] for details). However, on the one hand it is difficult to sample such a multi-dimensional parameter space sufficiently well, on the other hand it is well known that several of the parameters of the pMSSM-19 hardly affect Higgs phenomenology. We therefore focus in this paper on a smaller set of parameters, namely the phenomenological MSSM with the seven free parameters that we regard as most relevant for the phenomenology of Higgs and flavour physics (pMSSM-7, see below for details on these parameters). This seven-dimensional parameter space, which as we will demonstrate captures most of the allowed Higgs phenomenology of the MSSM, can be sampled quite well with $\mathcal{O}\left(10^{7}\right)$ scan points. We will comment in our analysis on the potential impact of varying further MSSM parameters.

In our analysis we perform fits in the MSSM both for the interpretation of the LHC signal in terms of the light and the heavy $\mathcal{C P}$-even Higgs of the MSSM and we compare the fit results with the SM case. We take into account all available individual search channels at ATLAS and CMS at 7 and $8 \mathrm{TeV}$ centre-of-mass energy that have been published by the end of July 2012, including also the two combined Higgs mass values. We furthermore include the final results of the Tevatron, corresponding to three additional channels. Besides the direct Higgs search channels we take into account the Higgs exclusion bounds, limits on the SUSY masses, as well as the most relevant set of low-energy observables, $\mathrm{BR}(b \rightarrow s \gamma), \mathrm{BR}\left(B_{s} \rightarrow \mu^{+} \mu^{-}\right), \mathrm{BR}\left(B_{u} \rightarrow \tau \nu_{\tau}\right),(g-2)_{\mu}$ and the mass of the $W$ boson, $M_{W}$.

The paper is organised as follows: Sect. 2 gives a summary of the most relevant supersymmetric sectors and parameters. In Sect. 3 we briefly review the calculations and codes used for the Higgs sector predictions, as well as for the low-energy observables. Details on the parameter scan are given. In Sect. 4 the results of the scan, the best-fit points and the preferred parameter regions are presented. We briefly discuss the origin of the most significant deviations of the pMSSM-7 predictions with respect to the SM results. The conclusions can be found in Sect. 5 .

\section{Theoretical framework for pMSSM-7}

In the following we briefly describe the relevant sectors of the MSSM and define the seven basic parameters for our scan. As a first and general simplification we restrict ourselves to the MSSM with real parameters. The restriction to seven free/independent parameters is based on the intention to sample the full parameter space with $\mathcal{O}\left(10^{7}\right)$ scan points, 
while ensuring that the most important effects in the MSSM Higgs sector and flavour physics are covered.

The tree-level values for the predictions of the MSSM Higgs sector quantities are determined by $\tan \beta$, the $\mathcal{C P}$-odd Higgs-boson mass $M_{A}$, and the $Z$ boson mass $M_{Z}$. These predictions include the other Higgs boson masses, their couplings to other MSSM particles, their production cross sections, as well as their decay properties. Consequently, we choose as free parameters the two tree-level parameters,

(i): $M_{A}$,

(ii): $\tan \beta$.
Beyond tree-level, the main correction to the Higgs boson masses stems from the $t / \tilde{t}$ sector, and for large values of $\tan \beta$ also from the $b / \tilde{b}$ sector. In order to fix our notations, we list the conventions for the input parameters from the scalar top and scalar bottom sector of the MSSM. The notation can be taken over to scalar taus and scalar tau neutrinos via the substitutions $\tilde{t} \rightarrow \tilde{v}_{\tau}, \tilde{b} \rightarrow \tilde{\tau}$, taking into account that the MSSM contains only the scalar superpartners of the lefthanded neutrinos. Furthermore, the same notation holds for the first and the second generation of scalar fermions. The mass matrices in the basis of the current eigenstates $\tilde{t}_{L}, \tilde{t}_{R}$ and $\tilde{b}_{L}, \tilde{b}_{R}$ are given by

$$
\begin{gathered}
\mathcal{M}_{\tilde{t}}^{2}=\left(\begin{array}{cc}
M_{\tilde{t}_{L}}^{2}+m_{t}^{2}+\cos 2 \beta\left(\frac{1}{2}-\frac{2}{3} s_{\mathrm{w}}^{2}\right) M_{Z}^{2} & m_{t} X_{t} \\
m_{t} X_{t} & M_{\tilde{t}_{R}}^{2}+m_{t}^{2}+\frac{2}{3} \cos 2 \beta s_{\mathrm{w}}^{2} M_{Z}^{2}
\end{array}\right), \\
\mathcal{M}_{\tilde{b}}^{2}=\left(\begin{array}{cc}
M_{\tilde{b}_{L}}^{2}+m_{b}^{2}+\cos 2 \beta\left(-\frac{1}{2}+\frac{1}{3} s_{\mathrm{w}}^{2}\right) M_{Z}^{2} & m_{b} X_{b} \\
m_{b} X_{b} & M_{\tilde{b}_{R}}^{2}+m_{b}^{2}-\frac{1}{3} \cos 2 \beta s_{\mathrm{w}}^{2} M_{Z}^{2}
\end{array}\right),
\end{gathered}
$$

where $M_{\tilde{t}_{L}}, M_{\tilde{t}_{R}}, M_{\tilde{b}_{L}}$, and $M_{\tilde{b}_{R}}$ are the soft SUSY-breaking mass parameters in the scalar top and bottom sector, $m_{t}$ and $m_{b}$ are the respective quark masses, $s_{\mathrm{W}}=\sqrt{1-M_{W}^{2} / M_{Z}^{2}}$ with $M_{W}$ denoting the mass of the $W$ boson, and

$m_{t} X_{t}=m_{t}\left(A_{t}-\mu \cot \beta\right), \quad m_{b} X_{b}=m_{b}\left(A_{b}-\mu \tan \beta\right)$.

Here $A_{t}\left(A_{b}\right)$ denotes the trilinear Higgs-stop (Higgssbottom) coupling. The higgsino mass parameter $\mu$, which also appears in Eq. (3), is taken as free scan parameter

(iii): $\mu$.

$\mathrm{SU}(2)$ gauge invariance leads to the relation $M_{\tilde{t}_{L}}=M_{\tilde{b}_{L}}$. To achieve an efficient sampling of the parameters, we have to limit the dimensionality of the parameter space under consideration. We therefore use a universal parameter also for the left/right-handed squark soft masses of the third generation (and similarly for the sleptons). As free parameters we choose

(iv): $\quad M_{\tilde{q}_{3}}:=M_{\tilde{t}_{L}}\left(=M_{\tilde{b}_{L}}\right)=M_{\tilde{t}_{R}}=M_{\tilde{b}_{R}}$,

(v): $\quad M_{\tilde{l}_{3}}:=M_{\tilde{\tau}_{L}}\left(=M_{\tilde{v}_{L}}\right)=M_{\tilde{\tau}_{R}}$,

(vi): $\quad A_{f}:=A_{t}=A_{b}=A_{\tau}$.

For the soft scalar masses of the first two generations, which are much less relevant for Higgs physics (but can play a rel- evant role for the low-energy observables), we choose fixed parameter values as

$M_{\tilde{q}_{L}}=M_{\tilde{q}_{R}}(q=c, s, u, d)=1000 \mathrm{GeV}$,

$M_{\tilde{l}_{L}}=M_{\tilde{l}_{R}}\left(l=\mu, v_{\mu}, e, v_{e}\right)=300 \mathrm{GeV}$.

The choice for the first and second generation squarks places their masses roughly at the level currently probed at the LHC. Somewhat larger values would have a minor impact on our analysis. The values for the first and second generation slepton mass parameters were chosen to provide rough agreement with the anomalous magnetic moment of the muon (see below).

The trilinear Higgs coupling parameter for the first two generations we set to

$A_{c, s, u, c, \mu, e}=A_{f}$.

The Higgs sector observables furthermore depend on the SU(2) gaugino mass parameter,

(vii): $M_{2}$,

which we take as the final free parameter in our analysis. The other electroweak gaugino mass parameter, $M_{1}$, is fixed via the GUT relation

$M_{1}=\frac{5}{3} \frac{s_{\mathrm{w}}^{2}}{c_{\mathrm{w}}^{2}} M_{2} \approx \frac{1}{2} M_{2}$. 
Table 1 Ranges used for the free parameters in the pMSSM-7 scan

\begin{tabular}{lll}
\hline Parameter & Minimum & Maximum \\
\hline$M_{A}[\mathrm{GeV}]$ & 90 & 1000 \\
$\tan \beta$ & 1 & 60 \\
$\mu[\mathrm{GeV}]$ & 200 & 3000 \\
$M_{\tilde{q}_{3}}[\mathrm{GeV}]$ & 200 & 1500 \\
$M_{\tilde{l}_{3}}[\mathrm{GeV}]$ & 200 & 1500 \\
$A_{f}[\mathrm{GeV}]$ & $-3 M_{\tilde{q}_{3}}$ & $3 M_{\tilde{q}_{3}}$ \\
$M_{2}[\mathrm{GeV}]$ & 200 & 500 \\
\hline
\end{tabular}

The gluino mass parameter, which enters the Higgs mass predictions only from two-loop order on, is fixed to a value close to the limits from recent searches at the LHC,

$M_{3}=m_{\tilde{g}}=1000 \mathrm{GeV}$.

An adjustment of the gluino mass parameter to even higher values is expected to have a negligible impact on our analysis.

\section{Model predictions and constraints}

\subsection{Parameter space scanning}

The pMSSM-7 parameter space is sampled by performing random scans (using uniform distributions) over the seven input parameters in the ranges given in Table 1 . The two cases, where either $h$ or $H$ corresponds to the signal at $\hat{M}_{H} \sim 126 \mathrm{GeV}$, are treated in two separate scans, and the results are discussed in parallel below. Each scan starts with $\mathcal{O}\left(10^{7}\right)$ randomly chosen points with a flat distribution over the parameter ranges. In the next step, we identify regions with a low $\chi^{2}$ and perform a dedicated, smaller, scan with $\mathcal{O}\left(10^{6}\right)$ points (again uniformly distributed, but in a more restricted range for some parameters) to improve the sampling efficiency in the interesting regions. ${ }^{1}$ In practice, the full parameter ranges from Table 1 are taken only for the light Higgs case, while for the heavy Higgs case we limit $M_{A}<200 \mathrm{GeV}$ and $\tan \beta<30$ (still using the full ranges for the other parameters), which improves the sampling efficiency in the relevant mass region for $M_{H}$. In addition to the free parameters listed in Table 1, the remaining parameters are fixed according to Sect. 2.

Since we are mainly interested in the Higgs sector, we do not exploit the full possibilities in the low-energy MSSM to vary the soft-breaking parameters of the first two generations or the gluino mass (we will comment below on a

\footnotetext{
${ }^{1}$ The reader should keep in mind here (and in the following) that the point density has no statistical meaning.
}

possible impact of this choice on the MSSM predictions for $\left.(g-2)_{\mu}\right)$. Consequently, it is not relevant to apply LHC exclusion bounds from supersymmetry searches in our analysis, since these can be avoided by adjusting the additional parameters to sufficiently high values with only small effects on the Higgs sector. We do, however, apply the results from Higgs boson searches, see the next subsection. We also apply the model-independent limits on sfermion and chargino masses, typically at the level of $\sim 100 \mathrm{GeV}$ from direct searches at LEP (as summarised in the PDG review [86]). Furthermore, we require that the lightest supersymmetric particle (LSP) is the lightest neutralino. The top quark pole mass is sampled from a Gaussian distribution with $m_{t}=173.2 \pm 0.9 \mathrm{GeV}$, using a cutoff at $\pm 2 \sigma$. Effects of other parametric uncertainties from SM quantities are estimated to be small, and are therefore neglected.

For the evaluation of the sparticle and Higgs masses we use the code FeynHiggs (version 2.9.4) [87-90]. The residual Higgs mass uncertainty from this calculation (e.g. from missing higher orders) is estimated to be around $2 \mathrm{GeV}$, depending on the considered region of parameter space [89]. We are interested in parameter points that give a Higgs mass prediction, for either $M_{h}$ or $M_{H}$, close to the observed LHC signal. We therefore constrain the analysis in a first step to points with $M_{h}$ or $M_{H}$ in the region 121-129 GeV. To avoid configurations in parameter space that give an unstable perturbative behaviour in the Higgs mass calculation, we use a criterion based on the $\mathbf{Z}$ matrix (as defined in [90]) and exclude points for which ||$Z_{k 1}^{2 \mathrm{~L}}|-| Z_{k 1}^{1 \mathrm{~L}}|| /\left|Z_{k 1}^{1 \mathrm{~L}}\right|>0.1$ (see [20] for a similar treatment). Here $k=1$ (2) is set for a SM-like light (heavy) Higgs, and the superscripts refer to the same quantity evaluated with 1-loop (1L) or 2-loop (2L) precision.

To obtain an indication of what the currently favoured regions of the MSSM parameter space are, we use a simple statistical treatment of the data where the different observables are taken into account by calculating, for every parameter point in the scan, a global $\chi^{2}$ function

$$
\begin{aligned}
\chi^{2}= & \sum_{i=1}^{n_{\mathrm{LHC}}} \frac{\left(\mu_{i}-\hat{\mu}_{i}\right)^{2}}{\sigma_{i}^{2}}+\sum_{i=1}^{n_{\mathrm{Tev}}} \frac{\left(\mu_{i}-\hat{\mu}_{i}\right)^{2}}{\sigma_{i}^{2}} \\
& +\frac{\left(M_{h, H}-\hat{M}_{H}\right)^{2}}{\sigma_{\hat{M}_{H}}^{2}}+\sum_{i=1}^{n_{\mathrm{LEO}}} \frac{\left(O_{i}-\hat{O}_{i}\right)^{2}}{\sigma_{i}^{2}} .
\end{aligned}
$$

Quantities with a hat denote experimental measurements, and unhatted quantities the corresponding model predictions for Higgs signal strength modifiers, $\mu_{i}$, and low-energy observables (LEO), $O_{i}$. The different observables entering Eq. (9) are described in more detail in the following sections. The combined uncertainties $\sigma_{i}$ contain the known theory and experimental uncertainties. Correlations are neglected throughout, since they are for most cases not publicly available. The total number of degrees of freedom, 
$v$, is counted in the naive way as $v=n_{\text {obs }}-n_{\text {para }}$, where $n_{\mathrm{obs}}=n_{\mathrm{LHC}}+n_{\mathrm{Tev}}+1+n_{\mathrm{LEO}}$ (for LHC, Tevatron, the Higgs boson mass, and low-energy observables); $n_{\text {para }}$ is the number of model parameters. In the SM we have $n_{\text {para }}=1$ (the Higgs mass), and for both MSSM analyses $n_{\text {para }}=7$.

\subsection{Direct Higgs searches}

We use two kinds of experimental data from direct Higgs searches in our analysis: exclusion limits at $95 \%$ confidence level (CL), and the signal rates measured in different channels for the observed LHC signal around $\hat{M}_{H} \sim 126 \mathrm{GeV}$. The exclusion limits from LEP, the Tevatron, and the LHC (including data published before ICHEP 2012) are taken into account using HiggsBounds version 3.8.0 [91, 92]. Since no $\chi^{2}$ information is available from these searches, but only the exclusion limits at a fixed level of confidence, we apply these limits as hard cuts on the parameter space. It should be noted, however, that HiggsBounds only tests for each parameter point the model predictions against the single channel with the highest expected sensitivity for an exclusion, in order to ensure a consistent interpretation of the exclusion limit as a $95 \% \mathrm{CL}$.

The measured signal strength modifiers, $\hat{\mu}_{i}$, for the observed Higgs-like state around $\hat{M}_{H} \sim 126 \mathrm{GeV}$ are taken into account in our fit directly in the $\chi^{2}$ evaluation (see Eq. (9)). The data for all included channels are given in Table 2, with the corresponding experimental signal strengths and their (asymmetric) $1 \sigma$ error bands. These rates provide the main dataset to which we fit the MSSM Higgs sector. In total we include 37 observables, where 34 are from the LHC experiments and 3 provide supplementary information from the Tevatron. The best fit signal strength modifiers of ATLAS and CMS are given for different Higgs masses, corresponding to the values measured by the individual experiments, i.e. we interpret the experimental discoveries as being compatible, and due to a single new state. The Tevatron data, which do not admit a mass measurement from the observed excess on its own, are evaluated for $\hat{M}_{H}=125 \mathrm{GeV}$. All values listed in Table 2 are extracted directly from the quoted experimental references, with one exception: ATLAS has not provided a measurement for the signal strength modifier of $H \rightarrow Z Z^{(*)}$ separately for the 7 and $8 \mathrm{TeV}$ data, but only for the combination (the $7 \mathrm{TeV}$ values are available from a previous analysis). To compare to our $8 \mathrm{TeV}$ predictions, these values are therefore calculated from the $7 \mathrm{TeV}$ and $7+8 \mathrm{TeV}$ data under the assumption of independent Gaussian measurements, following the procedure outlined in [52]. This should lead to an uncertainty on the estimated $8 \mathrm{TeV}$ rate of the same order as the overall uncertainty from neglecting the (unknown) correlations.
The MSSM predictions for the signal strength modifiers are evaluated according to

$\mu_{i}=\frac{\sum_{k} \omega_{i k} \sigma_{k}(p p \rightarrow h, H) \times \mathrm{BR}(h, H \rightarrow i)}{\sum_{k} \omega_{i k} \sigma_{k}^{\mathrm{SM}}(p p \rightarrow h, H) \times \mathrm{BR}^{\mathrm{SM}}(h, H \rightarrow i)}$,

where $\sigma_{k}(p p \rightarrow h, H)$ denotes the contribution to the Higgs production cross section from partonic subprocess $k$, evaluated at the predicted Higgs mass. The production modes considered are gluon-gluon fusion $(\mathrm{g} g)$, vector boson fusion (VBF), associated vector boson production (VH), and associated $t \bar{t} h(H)$ production. The experimental efficiencies $\omega_{i k}$ have only been published by ATLAS and CMS for the $\gamma \gamma$ analysis; for CMS in the case of the subcategories, and for ATLAS also the inclusive result. We make use of these numbers when they are available. For all other channels we have to use the "naive" efficiencies deducible from the analysis description (e.g. for a VBF-type analysis tagging two forward jets, we set $\omega=1$ for the VBF cross section, whereas all other modes have $\omega=0$ ). In channels where the mass resolution is not good enough to separate contributions from different Higgs bosons, we approximate the contributions from $H$ and the $\mathcal{C P}$-odd Higgs $A$ by adding their signal rates incoherently. We do not add the rates of the $\mathcal{C P}$-even Higgs bosons, whose joint contributions to the signal could also include interference effects. Our analysis is therefore limited to the case with a single $\mathcal{C} \mathcal{P}$-even Higgs boson close to the observed signal, and we leave a more detailed treatment of the case when $M_{h} \sim M_{H} \sim 126 \mathrm{GeV}$ to a dedicated analysis. Since the $\mathcal{C P}$-odd Higgs does not have tree-level couplings to vector bosons (and hence also a reduced coupling to photons), it gives a negligible contribution to the channels with vector bosons in the Higgs production and/or decay. Effectively, the $\mathcal{C P}$-odd Higgs therefore only plays a role for the inclusive $(0 / 1$ jet $) \tau^{+} \tau^{-}$channels. In these channels it can easily dominate over the $H$ contribution for large values of $\tan \beta$. In the light Higgs case, we find that the masses of $h$ and $A$ differ by $M_{A}-M_{h} \gtrsim 50 \mathrm{GeV}$ in the favoured region (see below). Thus we do not take any contributions to the $h$ rates from the $\mathcal{C P}$-odd Higgs into account.

The cross section predictions entering Eq. (10) are calculated, both in the MSSM and the SM, using FeynHiggs (version 2.9.4) [87-90]. For the SM cross sections the results of the LHC Higgs cross section working group are implemented [97-99] (where the $g g$ production cross sections are taken from $[100,101])$. The corresponding MSSM production cross-sections are obtained in the effective-coupling approximation [102]. The $g g$ production cross section follows the description in [103], where results of [104-107] were used. The decay width evaluation includes a full oneloop correction for the decay to fermions [108, 109]; see [103] for more details on the other channels.

In addition to the signal strength modifiers, we include a $\chi^{2}$ contribution from the measured Higgs mass $\hat{M}_{H}$ (which 
Table 2 Experimentally measured values for the Higgs signal strength modifiers, and their corresponding uncertainties (lower/upper edges of $1 \sigma$ error bars), in the various channels

\begin{tabular}{|c|c|c|c|c|c|}
\hline Channel & $\sqrt{s}[\mathrm{TeV}]$ & $\hat{\mu}_{\text {low }}$ & $\hat{\mu}$ & $\hat{\mu}_{\text {up }}$ & Reference \\
\hline \multicolumn{6}{|c|}{ ATLAS data at $\hat{M}_{H}=126.5 \mathrm{GeV}$} \\
\hline$b \bar{b}$ & 7 & -1.646 & 0.510 & 2.680 & {$[6]$} \\
\hline$\tau \tau$ & 7 & -1.550 & 0.464 & 2.011 & {$[6]$} \\
\hline$W W$ & 7 & -0.164 & 0.438 & 1.103 & [93] \\
\hline$W W$ & 8 & 1.308 & 1.920 & 2.536 & [94] \\
\hline$\gamma \gamma$ (inclusive) & 7 & 1.397 & 2.155 & 2.903 & [4] \\
\hline$\gamma \gamma$ (inclusive) & 8 & 1.054 & 1.685 & 2.326 & {$[4]$} \\
\hline$Z Z$ & 7 & 0.405 & 1.080 & 2.177 & {$[95]$} \\
\hline$Z Z$ & 8 & 0.400 & 1.049 & 1.708 & [96] \\
\hline \multicolumn{6}{|c|}{ CMS data at $\hat{M}_{H}=125.0 \mathrm{GeV}$} \\
\hline$b \bar{b}(\mathrm{VH})$ & 7 & -0.606 & 0.588 & 1.824 & [7] \\
\hline$b \bar{b}(\mathrm{VH})$ & 8 & -0.441 & 0.424 & 1.535 & [7] \\
\hline$b \bar{b}(t \bar{t} H)$ & 7 & -2.624 & -0.771 & 1.288 & [7] \\
\hline$\tau \tau(0 / 1$ jet $)$ & 7 & -0.400 & 1.000 & 2.441 & [7] \\
\hline$\tau \tau(0 / 1$ jet $)$ & 8 & 0.588 & 2.153 & 3.635 & [7] \\
\hline$\tau \tau(\mathrm{VBF})$ & 7 & -2.912 & -1.718 & -0.359 & [7] \\
\hline$\tau \tau(\mathrm{VBF})$ & 8 & -3.035 & -1.759 & -0.400 & [7] \\
\hline$\tau \tau(\mathrm{VH})$ & 7 & -2.418 & 0.671 & 4.788 & [7] \\
\hline$\gamma \gamma$ (Dijet loose) & 8 & -2.660 & -0.626 & 1.409 & {$[5]$} \\
\hline$\gamma \gamma$ (Dijet tight) & 8 & -0.267 & 1.289 & 2.868 & {$[5]$} \\
\hline$\gamma \gamma$ (Untagged 3) & 8 & 2.007 & 3.754 & 5.549 & {$[5]$} \\
\hline$\gamma \gamma$ (Untagged 2) & 8 & -0.195 & 0.930 & 2.080 & {$[5]$} \\
\hline$\gamma \gamma$ (Untagged 1) & 8 & 0.475 & 1.504 & 2.533 & {$[5]$} \\
\hline$\gamma \gamma($ Untagged 0$)$ & 8 & 0.212 & 1.456 & 2.701 & [5] \\
\hline$\gamma \gamma($ Dijet $)$ & 7 & 2.174 & 4.209 & 6.243 & [5] \\
\hline$\gamma \gamma$ (Untagged 3) & 7 & -0.099 & 1.528 & 3.132 & {$[5]$} \\
\hline$\gamma \gamma($ Untagged 2) & 7 & -0.434 & 0.715 & 1.887 & {$[5]$} \\
\hline$\gamma \gamma$ (Untagged 1) & 7 & -0.291 & 0.643 & 1.600 & {$[5]$} \\
\hline$\gamma \gamma($ Untagged 0$)$ & 7 & 1.337 & 3.132 & 4.974 & {$[5]$} \\
\hline$W W(0 / 1$ jet $)$ & 7 & -0.029 & 0.588 & 1.206 & [7] \\
\hline$W W(0 / 1$ jet $)$ & 8 & 0.176 & 0.835 & 1.494 & {$[7]$} \\
\hline$W W(\mathrm{VBF})$ & 7 & -3.900 & -1.306 & 0.918 & [7] \\
\hline$W W(\mathrm{VBF})$ & 8 & -0.523 & 1.371 & 3.347 & [7] \\
\hline$W W(\mathrm{VH})$ & 7 & -5.753 & -2.829 & 0.341 & [7] \\
\hline$Z Z$ & 7 & 0.176 & 0.671 & 1.371 & [7] \\
\hline$Z Z$ & 8 & 0.259 & 0.794 & 1.494 & [7] \\
\hline \multicolumn{6}{|c|}{ Tevatron data at $\hat{M}_{H}=125.0 \mathrm{GeV}$} \\
\hline$b \bar{b}$ & 1.96 & 1.290 & 1.970 & 2.710 & {$[3]$} \\
\hline$\gamma \gamma$ & 1.96 & 1.080 & 3.620 & 6.580 & {$[3]$} \\
\hline$W W$ & 1.96 & 0.000 & 0.320 & 1.450 & {$[3]$} \\
\hline
\end{tabular}

in the MSSM can correspond to either $M_{h}$ or $M_{H}$ ). Unlike in the SM, $M_{h}$ and $M_{H}$ are not free parameters in the MSSM but a prediction of the theory. The Higgs mass observable is included here on the same footing as the measured rates. There are a number of other available studies (see, for example, [19, 21-24]) showing the constraints on the MSSM parameter space from the Higgs mass alone.
Averaging the ATLAS and CMS measurements, we obtain $\hat{M}_{H}=125.7 \mathrm{GeV}$. To fully cover the difference between the individual experimental measurements we assign a conservative uncertainty of $\sigma_{\hat{M}_{H}}^{\exp }=1 \mathrm{GeV}$. With more data we expect this experimental uncertainty on the Higgs mass to be significantly reduced. In addition, we add linearly to this number a theoretical uncertainty $\sigma_{\hat{M}_{H}}^{\text {theo }}=2 \mathrm{GeV}$ [89], which 
Table 3 The experimental values and (SM) theory predictions for low-energy observables (LEO) used to constrain the MSSM parameter space

\begin{tabular}{llll}
\hline Observable & Experimental value & & SM value \\
\hline $\mathrm{BR}\left(B \rightarrow X_{s} \gamma\right)$ & $(3.43 \pm 0.21 \pm 0.07) \times 10^{-4}$ & {$[127]$} & $(3.08 \pm 0.22) \times 10^{-4}$ \\
$\mathrm{BR}\left(B_{s} \rightarrow \mu^{+} \mu^{-}\right)$ & $<4.2 \times 10^{-9}$ & {$[128-130]$} & $(3.55 \pm 0.38) \times 10^{-9}$ \\
$\mathrm{BR}\left(B_{u} \rightarrow \tau \nu_{\tau}\right)$ & $(1.66 \pm 0.33) \times 10^{-4}$ & {$[127]$} & $(1.01 \pm 0.29) \times 10^{-4}$ \\
$\delta a_{\mu}$ & $(30.2 \pm 9.0) \times 10^{-10}$ & {$[131-133]$} & - \\
$M_{W}$ & $(80.385 \pm 0.015) \mathrm{GeV}$ & {$[120,121]$} & $(80.363 \pm 0.004) \mathrm{GeV}$ \\
\hline
\end{tabular}

Table 4 Global $\chi^{2}$ results with $v$ degrees of freedom from the fits of the SM and the MSSM with either $h$ or $H$ as the LHC signal, the reduced $\chi_{v}^{2} \equiv \chi^{2} / \nu$, and the corresponding $p$-values. The number of degrees of freedom are evaluated naively as $v=n_{\mathrm{obs}}-n_{\text {param }}$

\begin{tabular}{|c|c|c|c|c|c|c|c|c|c|c|c|c|}
\hline \multirow[t]{2}{*}{ Case } & \multicolumn{3}{|c|}{ LHC only } & \multicolumn{3}{|c|}{ LHC+Tevatron } & \multicolumn{3}{|c|}{$\mathrm{LHC}+\mathrm{LEO}$} & \multicolumn{3}{|c|}{ LHC+Tevatron+LEO } \\
\hline & $\chi^{2} / v$ & $\chi_{v}^{2}$ & $p$ & $\overline{\chi^{2} / v}$ & $\chi_{v}^{2}$ & $p$ & $\chi^{2} / v$ & $\chi_{v}^{2}$ & $p$ & $\chi^{2} / v$ & $\chi_{v}^{2}$ & $p$ \\
\hline SM & $27.6 / 34$ & 0.81 & 0.77 & $31.0 / 37$ & 0.84 & 0.74 & $41.6 / 39$ & 1.07 & 0.36 & $45.3 / 42$ & 1.08 & 0.34 \\
\hline$h$ & $23.3 / 28$ & 0.83 & 0.72 & $26.8 / 31$ & 0.86 & 0.68 & $26.7 / 33$ & 0.81 & 0.77 & $30.4 / 36$ & 0.84 & 0.73 \\
\hline$H$ & $26.0 / 28$ & 0.93 & 0.57 & $33.1 / 31$ & 1.07 & 0.37 & $35.5 / 33$ & 1.08 & 0.35 & $42.4 / 36$ & 1.18 & 0.21 \\
\hline
\end{tabular}

accounts for the uncertainty in the MSSM Higgs mass calculation. The total uncertainty entering Eq. (9) is therefore $\sigma_{\hat{M}_{H}}=3 \mathrm{GeV} .{ }^{2}$ We note that, for a Higgs mass in the considered range $\left(121 \mathrm{GeV}<M_{h}<129 \mathrm{GeV}\right)$, this uncertainty results in a maximal $\chi^{2}$ contribution of $\Delta \chi^{2}=2.4$ from the Higgs mass observable.

\subsection{Low-energy observables}

In addition to the measurements related to the LHC Higgs signal, we include several low-energy observables (LEO) in the fit. These are listed in Table 3, which summarises the experimental values $^{3}$ (for the case of $\operatorname{BR}\left(B_{s} \rightarrow \mu^{+} \mu^{-}\right)$the upper limit) and the corresponding SM theory predictions (evaluated for $M_{H}^{\mathrm{SM}}=125.7 \mathrm{GeV}$ and $m_{t}=173.2 \mathrm{GeV}$ ). The flavour physics observables are evaluated (both in the SM and the MSSM) using SuperIso (version 3.2) [111$113]$, which in particular contains the results for $\mathrm{BR}(B \rightarrow$ $\left.X_{s} \gamma\right)$ based on the NNLO calculation of [114]. Our fit includes also the anomalous magnetic moment of the muon, $a_{\mu}=\frac{1}{2}(g-2)_{\mu}$, which shows a deviation of more than $3 \sigma$ between the experimental measurement and the SM predic-

\footnotetext{
${ }^{2}$ If the experimental and theory uncertainties were instead combined in quadrature, this would give a total Higgs mass uncertainty of $\sigma_{\hat{M}_{H}}=$ $2.24 \mathrm{GeV}$. Such a change would have a negligible impact on the overall result.

${ }^{3}$ We note that the Belle Collaboration has recently reported a new measurement of $\operatorname{BR}\left(B_{u} \rightarrow \tau v_{\tau}\right)$, which is in better agreement with the SM (and thus naturally with models with two Higgs doublets) [110]. While we do not take this new result into account in our overall fit results, we do comment briefly on its possible effects.
}

tion. ${ }^{4}$ We use SuperIso to calculate the MSSM contribution $\delta a_{\mu}$ to the anomalous magnetic moment of the muon (we have cross-checked those results with FeynHiggs and found good agreement) including the dominant two-loop contributions [116-118], see [119] for a review.

As a final observable we also include the MSSM prediction of the $W$ boson mass into the fit. Here the SM prediction shows a $\sim 1.5 \sigma$ deviation from the latest experimental value, $M_{W}^{\exp }=80.385 \pm 0.015 \mathrm{GeV}[120,121]$. Our MSSM evaluation of $M_{W}$ is done using FeynHiggs, where the full SM result [122] is supplemented with the leading corrections from the $\tilde{t} / \tilde{b}$ sector $[18,123,124]$. A comparison with the best available MSSM evaluation [125, 126] shows that corrections larger than $10 \mathrm{MeV}$ can be missed if some uncoloured SUSY particles are light. Consequently, we assign a theory uncertainty of $15 \mathrm{MeV}$ to our $M_{W}$ evaluation and conservatively combine it with the experimental uncertainty linearly. Thus in total we take an uncertainty of $\pm 30 \mathrm{MeV}$ into account.

\section{Results}

In Table 4 we present the results of our fits in terms of total $\chi^{2}$ values (with $v$ degrees of freedom), the reduced $\chi_{v}^{2} \equiv$ $\chi^{2} / v$, and the corresponding $p$-values. Since $v$ is derived via the naive counting, the absolute numbers of the $p$-values should not be overinterpreted; the relative numbers, however, give a good impression of the relative goodness of the fits. For each MSSM interpretation (the cases of either $h$ or $H$ as the $125.7 \mathrm{GeV}$ signal) we present four different fits:

\footnotetext{
${ }^{4}$ The most recent evaluation of $a_{\mu}$ in the SM [115], taking also $\tau$ data into account, finds an even larger deviation of more than $4 \sigma$.
} 
Fig. 1 Fit results for the signal strength modifiers, $\mu_{i}$, in the case that the light $\mathcal{C} \mathcal{P}$-even Higgs is interpreted as the new boson around $\sim 125.7 \mathrm{GeV}$ ("light Higgs case"). The experimental data are shown as black dots (with error bars). The other symbols show best fit points, corresponding to the full fit (LHC+Tevatron+LEO) (blue solid squares), without the Tevatron data (blue open squares), and without LEO (red diamonds) (Color figure online)

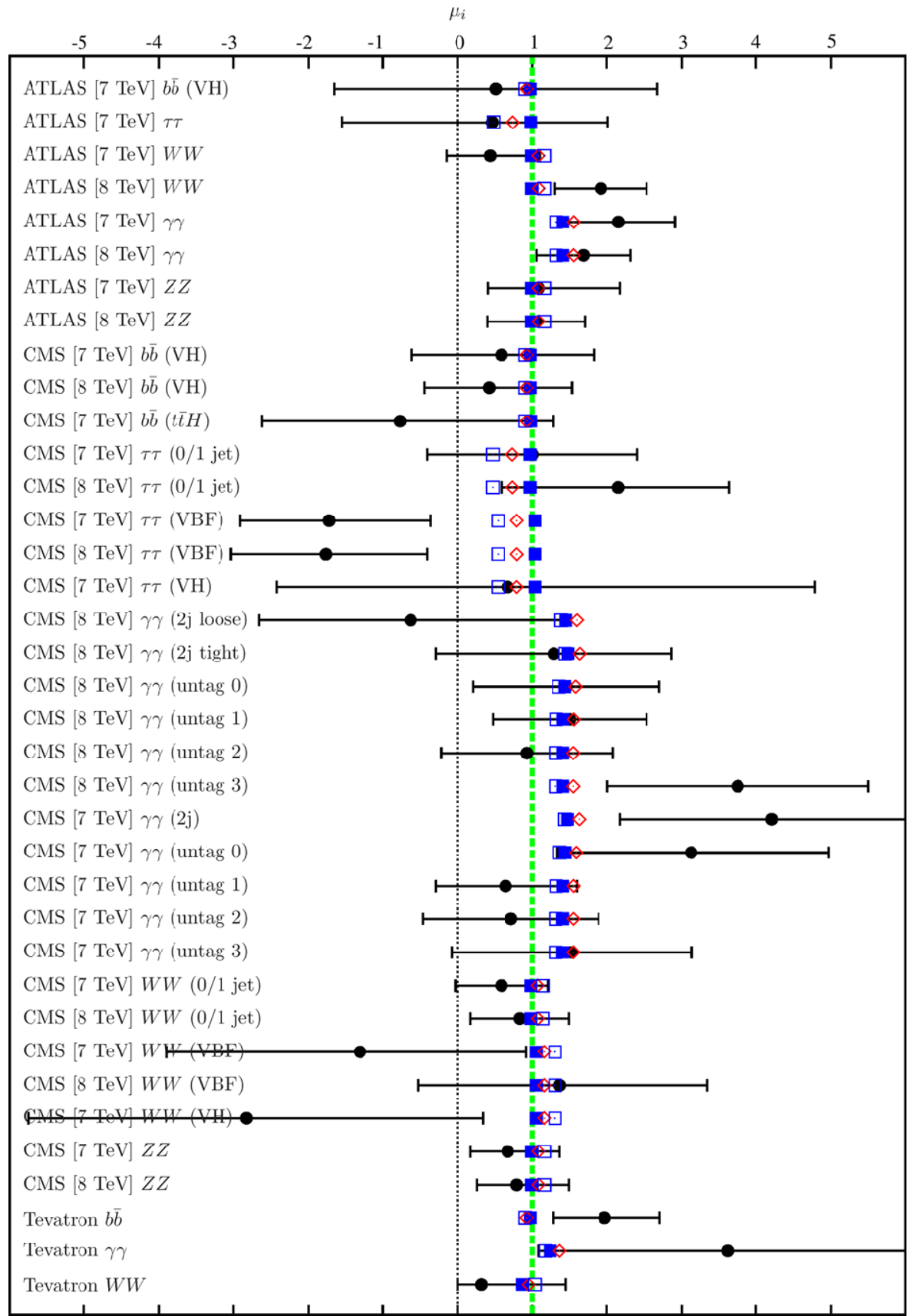

one taking the complete dataset (LHC+Tevatron+LEO) into account, one where the low-energy observables (LEO) are left out, one where the Tevatron data are left out, and finally the fit where only LHC observables are considered. When the fit is performed using only the high-energy collider data, both with and without the Tevatron results, the obtained $\chi^{2}$ values of the best fit points are quite similar between the SM and the two MSSM interpretations, where the fit in the heavy Higgs case becomes slightly worse after the inclusion of the Tevatron data. When low energy observables are included, the SM and the heavy Higgs case fits become somewhat worse. In the latter case this can be understood from the potentially larger contributions of light Higgs bosons to $B$-physics observables. For the SM fit the reason lies in the fact that the SM prediction for $(g-2)_{\mu}$ differs by more than $3 \sigma$ from the experimental value. Still we find that the SM provides a good fit to the full dataset, with $p_{\mathrm{SM}}=0.34$. On the other hand, concerning the MSSM it should be kept in mind that we did not fit the second generation slepton masses, which could potentially further improve the $a_{\mu}$ fit. For the complete fit, the corresponding $p$-values in the MSSM cases are $p_{h}=0.73\left(p_{H}=0.21\right)$ for the $h$ $(H)$ interpretations, respectively, which are both acceptable $p$-values. Overall, the data shows no clear preference for the 
Fig. 2 Fit results for the signal strength modifiers, $\mu_{i}$, in the case that the heavy $\mathcal{C P}$-even Higgs is interpreted as the new boson around $\sim 125.7 \mathrm{GeV}$ ("heavy Higgs case"). The experimental data are shown as black dots (with error bars). The other symbols show best fit points, corresponding to the full fit (LHC+Tevatron+LEO) (blue solid squares), without the Tevatron data (blue open squares), and without LEO (red diamonds) (Color figure online)

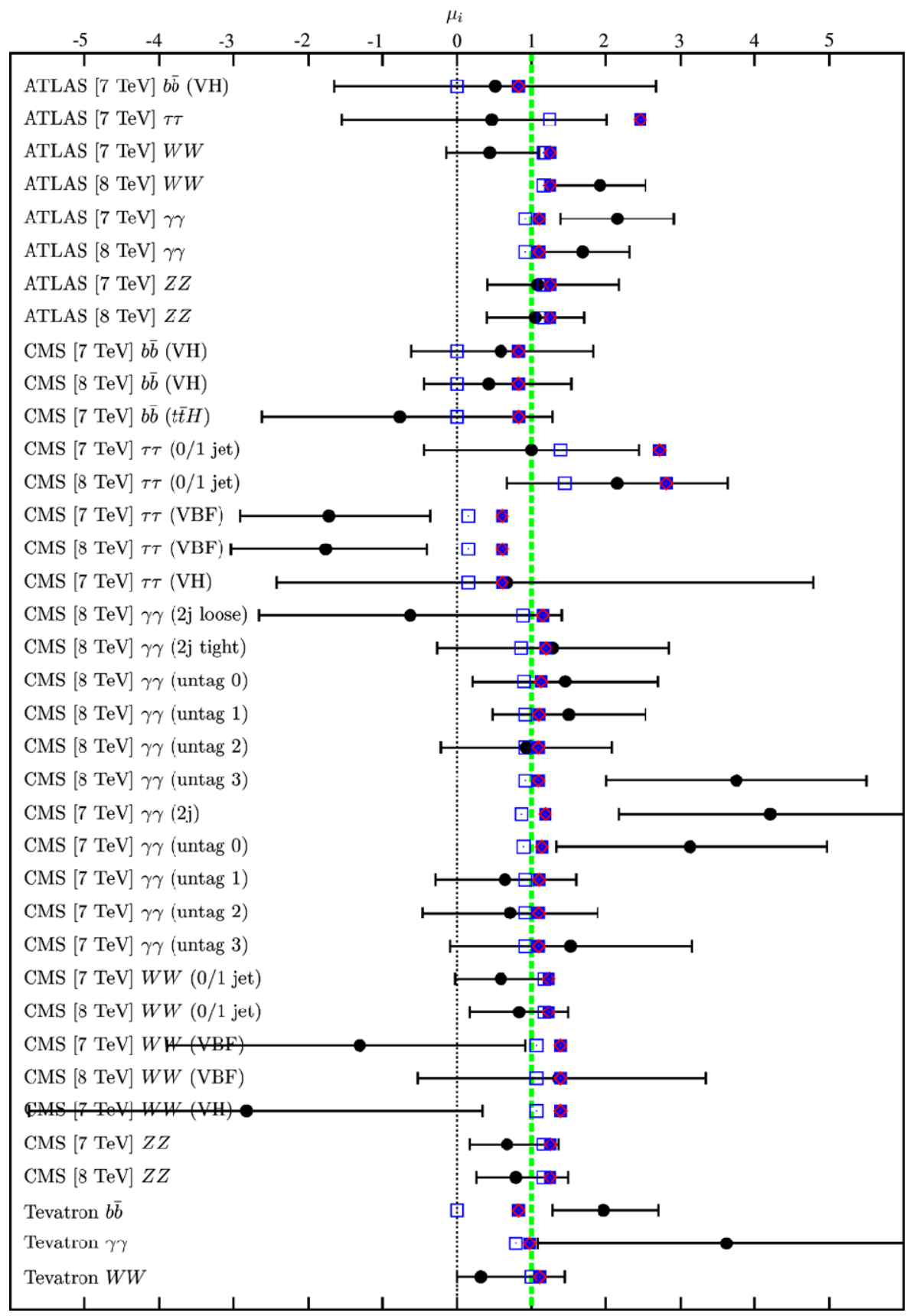

MSSM over the SM at this point. While the MSSM fits, in particular in the light Higgs case, yield lower $\chi^{2}$ values than the SM, this comes at the expense of additional parameters, so that the difference in the $p$-values is rather moderate. It is interesting to note that the fit to the heavy Higgs case, while not being as good as in the light Higgs case, still provides an acceptable description of all the data, i.e. the MSSM in the non-decoupling region still provides a viable solution. This interesting case will be analysed in more detail below.

Starting with the best fit for the $h$ case, we show in Fig. 1 the different best fit points using all available data (LHC, Tevatron, LEO) (blue solid squares), leaving out LEO (red diamonds) or leaving out the Tevatron data (blue open squares). The comparison of these three different types of result allows to trace the origin of trends in the fitted parameters. The experimental data on the signal strength modifiers in the different channels (as indicated in the figure) is shown as black dots, with the error bars corresponding to $\pm 1 \sigma$ uncertainties on $\hat{\mu}$. The values for the best fit point of the complete fit (LHC, Tevatron, LEO) are also presented in tabular form in Table 5. From here we can determine some characteristics of the best fit point, such as a significantly enhanced rate in the $\gamma \gamma$ final state and nearly SM rates for the other channels. Leaving out the Tevatron data a (small) suppres- 
Table 5 Best fit results (for the complete fit) with corresponding $\chi^{2}$ contributions and pulls for each observable

\begin{tabular}{|c|c|c|c|c|c|c|c|c|}
\hline Channel & & $\sqrt{s}[\mathrm{TeV}]$ & $\mu_{h}$ & $\chi_{h}^{2}$ & Pull & $\mu_{H}$ & $\chi_{H}^{2}$ & Pull \\
\hline ATLAS & $b \bar{b}$ & 7 & 0.98 & 0.05 & 0.22 & 0.83 & 0.02 & 0.15 \\
\hline ATLAS & $\tau \tau$ & 7 & 0.98 & 0.11 & 0.33 & 2.46 & 1.67 & 1.29 \\
\hline ATLAS & $W W$ & 7 & 0.99 & 0.69 & 0.83 & 1.25 & 1.50 & 1.22 \\
\hline ATLAS & $W W$ & 8 & 0.99 & 2.31 & -1.52 & 1.25 & 1.19 & -1.09 \\
\hline ATLAS & $\gamma \gamma$ & 7 & 1.41 & 0.95 & -0.98 & 1.10 & 1.94 & -1.39 \\
\hline ATLAS & $\gamma \gamma$ & 8 & 1.42 & 0.18 & -0.43 & 1.10 & 0.87 & -0.93 \\
\hline ATLAS & $Z Z$ & 7 & 0.99 & 0.02 & -0.13 & 1.25 & 0.02 & 0.16 \\
\hline ATLAS & $Z Z$ & 8 & 0.99 & 0.01 & -0.09 & 1.25 & 0.09 & 0.31 \\
\hline CMS & $b \bar{b}(\mathrm{VH})$ & 7 & 0.98 & 0.10 & 0.32 & 0.83 & 0.04 & 0.19 \\
\hline CMS & $b \bar{b}(\mathrm{VH})$ & 8 & 0.98 & 0.25 & 0.50 & 0.83 & 0.13 & 0.36 \\
\hline CMS & $b \bar{b}(\mathrm{ttH})$ & 7 & 0.98 & 0.72 & 0.85 & 0.83 & 0.61 & 0.78 \\
\hline CMS & $\tau \tau(0 / 1$ jets $)$ & 7 & 0.97 & 0.00 & -0.02 & 2.72 & 1.43 & 1.20 \\
\hline CMS & $\tau \tau(0 / 1$ jets $)$ & 8 & 0.97 & 0.57 & -0.76 & 2.81 & 0.20 & 0.44 \\
\hline CMS & $\tau \tau(\mathrm{VBF})$ & 7 & 1.04 & 4.12 & 2.03 & 0.61 & 2.92 & 1.71 \\
\hline CMS & $\tau \tau(\mathrm{VBF})$ & 8 & 1.04 & 4.24 & 2.06 & 0.61 & 3.03 & 1.74 \\
\hline CMS & $\tau \tau(\mathrm{VH})$ & 7 & 1.04 & 0.01 & 0.09 & 0.61 & 0.00 & -0.02 \\
\hline CMS & $\gamma \gamma$ (Dijet loose) & 8 & 1.45 & 1.04 & 1.02 & 1.15 & 0.76 & 0.87 \\
\hline CMS & $\gamma \gamma($ Dijet tight $)$ & 8 & 1.48 & 0.01 & 0.12 & 1.19 & 0.00 & -0.06 \\
\hline CMS & $\gamma \gamma($ Untagged 0$)$ & 8 & 1.44 & 0.00 & -0.02 & 1.13 & 0.07 & -0.26 \\
\hline CMS & $\gamma \gamma($ Untagged 1) & 8 & 1.42 & 0.01 & -0.09 & 1.10 & 0.16 & -0.39 \\
\hline CMS & $\gamma \gamma($ Untagged 2) & 8 & 1.41 & 0.18 & 0.42 & 1.09 & 0.02 & 0.14 \\
\hline CMS & $\gamma \gamma($ Untagged 3$)$ & 8 & 1.41 & 1.80 & -1.34 & 1.09 & 2.32 & -1.52 \\
\hline CMS & $\gamma \gamma$ (Dijet) & 7 & 1.48 & 1.80 & -1.34 & 1.19 & 2.21 & -1.49 \\
\hline CMS & $\gamma \gamma($ Untagged 0$)$ & 7 & 1.44 & 0.89 & -0.94 & 1.14 & 1.24 & -1.11 \\
\hline CMS & $\gamma \gamma($ Untagged 1) & 7 & 1.41 & 0.65 & 0.81 & 1.10 & 0.23 & 0.48 \\
\hline CMS & $\gamma \gamma($ Untagged 2) & 7 & 1.41 & 0.35 & 0.59 & 1.09 & 0.10 & 0.32 \\
\hline CMS & $\gamma \gamma$ (Untagged 3) & 7 & 1.41 & 0.01 & -0.07 & 1.09 & 0.07 & -0.27 \\
\hline CMS & $W W(0 / 1$ jets $)$ & 7 & 0.98 & 0.40 & 0.64 & 1.23 & 1.09 & 1.04 \\
\hline CMS & $W W(0 / 1$ jets $)$ & 8 & 0.98 & 0.05 & 0.22 & 1.23 & 0.36 & 0.60 \\
\hline CMS & $W W(\mathrm{VBF})$ & 7 & 1.05 & 1.12 & 1.06 & 1.39 & 1.47 & 1.21 \\
\hline CMS & $W W(\mathrm{VBF})$ & 8 & 1.05 & 0.03 & -0.17 & 1.39 & 0.00 & 0.01 \\
\hline CMS & $W W(\mathrm{VH})$ & 7 & 1.05 & 1.50 & 1.22 & 1.39 & 1.78 & 1.33 \\
\hline CMS & $Z Z$ & 7 & 0.99 & 0.21 & 0.45 & 1.25 & 0.69 & 0.83 \\
\hline CMS & $Z Z$ & 8 & 0.99 & 0.08 & 0.28 & 1.25 & 0.43 & 0.65 \\
\hline LHC & Higgs mass [GeV] & & 126.1 & 0.02 & 0.13 & 125.8 & 0.00 & 0.03 \\
\hline Tevatron & $b \bar{b}$ & 1.96 & 0.98 & 2.13 & -1.46 & 0.83 & 2.82 & -1.68 \\
\hline Tevatron & $\gamma \gamma$ & 1.96 & 1.24 & 0.88 & -0.94 & 0.97 & 1.08 & -1.04 \\
\hline Tevatron & $W W$ & 1.96 & 0.87 & 0.24 & 0.49 & 1.11 & 0.49 & 0.70 \\
\hline LEO & \multicolumn{2}{|l|}{$\mathrm{BR}\left(B \rightarrow X_{s} \gamma\right) \times 10^{4}$} & 3.41 & 0.00 & -0.03 & 4.38 & 2.12 & 1.46 \\
\hline LEO & \multicolumn{2}{|l|}{$\mathrm{BR}\left(B_{s} \rightarrow \mu^{+} \mu^{-}\right) \times 10^{9}$} & 2.79 & 0.00 & 0.00 & 2.24 & 0.00 & 0.00 \\
\hline LEO & \multicolumn{2}{|l|}{$\mathrm{BR}\left(B_{u} \rightarrow \tau \nu_{\tau}\right) \times 10^{4}$} & 0.98 & 2.37 & -1.54 & 0.80 & 3.78 & -1.94 \\
\hline LEO & \multicolumn{2}{|l|}{$\delta a_{\mu} \times 10^{9}$} & 2.58 & 0.24 & -0.49 & 1.34 & 3.48 & -1.87 \\
\hline LEO & \multicolumn{2}{|l|}{$M_{W}[\mathrm{GeV}]$} & 80.379 & 0.04 & -0.19 & 80.383 & 0.00 & -0.05 \\
\hline
\end{tabular}


sion of the fermionic final states can be observed. The fitted rates demonstrate that the pMSSM-7 is able to accommodate the main trends in the LHC/Tevatron data. Comparing the best fit points with/without LEO, we find a qualitatively very similar behaviour.

In Table 5 we give the details on the results for the lowenergy observables. In the light Higgs case, the only relevant contribution to the total $\chi^{2}$ comes from $\operatorname{BR}\left(B_{u} \rightarrow \tau \nu_{\tau}\right)$. Using an experimental value close to the new Belle result [110] would substantially reduce this $\chi^{2}$ contribution and lead to an even better fit. The best-fit value of $\operatorname{BR}\left(B_{s} \rightarrow \mu^{+} \mu^{-}\right)$ lies below the SM prediction. This feature is indeed found for most of our favoured region. We have checked that this trend is present already without taking the $\chi^{2}$ contribution of $\mathrm{BR}\left(B_{s} \rightarrow \mu^{+} \mu^{-}\right)$itself into account, see also the discussion in [50].

The best fit points for the heavy Higgs case are presented in Fig. 2 (numerical values in Table 5). As the figure shows, the same best fit point (albeit with different total $\chi^{2}$ ) is obtained for the different cases with/without LEO. Leaving out the Tevatron data, however, has a larger qualitative impact on the results, and rates close to zero are allowed in the $b \bar{b}$ channel, which will be discussed below in more detail. Since the alternative hypothesis of a heavy Higgs explanation for the LHC signal is fitted to the same data as for the $h$ case, and we have already seen that the overall $\chi^{2}$ is similar to (although slightly higher than) the light Higgs case, it is perhaps not so surprising to find that comparable rates are obtained for the best fit in the heavy Higgs case. This illustrates again that this interpretation is also a possible scenario to explain the LHC Higgs signal in the MSSM. The more indepth results below are therefore presented in parallel for the two separate cases with $h$ or $H$ corresponding to the signal discovered at the LHC.

In Table 5 we also give the results for the low-energy observables in the heavy Higgs case. One can see that the relatively small value of the Higgs mass scale in this case leads to non-negligible $\chi^{2}$ contributions from $\mathrm{BR}\left(B \rightarrow X_{s} \gamma\right)$ and $\operatorname{BR}\left(B_{u} \rightarrow \tau v_{\tau}\right)$, where the latter would substantially improve for a value close to the new Belle result. Also the SUSY contribution to $a_{\mu}$ turns out to be relatively small, giving a sizable contribution to the total $\chi^{2}$ (which is, however, affected by our choice to keep the slepton mass parameters fixed). Concerning $\operatorname{BR}\left(B_{s} \rightarrow \mu^{+} \mu^{-}\right)$it should be noted that, as in the light Higgs case, the preferred value is below the SM result, which again holds for most of the favoured region.

We now turn from the global fit properties and the best fit points to a more detailed analysis of the scan results. Figure 3 shows distributions of $\Delta \chi_{h}^{2}=\chi_{h}^{2}-\chi_{h, \text { min }}^{2}$ (light Higgs case) for the different signal rates. The colour coding is as follows: all points analysed in the scan (which pass theoretical consistency checks and have one $\mathcal{C P}$-even
Higgs boson in the interval $121 \mathrm{GeV}<M_{h}<129 \mathrm{GeV}$ ) are shown in grey. The blue points in addition fulfill constraints at $95 \%$ CL from direct Higgs searches applied by HiggsBounds 3.8.0. The three rows show the results for the full fit (upper row), excluding LEO (middle row), and excluding the Tevatron data (lower row). The signal rates are calculated as the inclusive Higgs production cross section (evaluated at $\sqrt{s}=8 \mathrm{TeV}$ ) times the decay rate, normalised to the SM predictions

$$
R_{X}^{h, H}=\frac{\sum_{i} \sigma_{i}(p p \rightarrow h, H) \times \mathrm{BR}(h, H \rightarrow X)}{\sum_{i} \sigma_{i}^{\mathrm{SM}}(p p \rightarrow h, H) \times \mathrm{BR}^{\mathrm{SM}}(h, H \rightarrow X)} .
$$

The only final state for which we consider a different observable than the fully inclusive Higgs production is $b \bar{b}$, where the sum is only taken over the cross sections for $(h, H) Z$ and $(h, H) W^{ \pm}$associated production. As described above, for the inclusive $\tau^{+} \tau^{-}$channels we consider the contribution of both $H$ and $A$ when these are close in mass. To make it clear when this is the case, we denote the joint (inclusive) rate as $R_{\tau \tau}^{H / A}$. We also define a common rate for vector boson final states $R_{V V}:=R_{W W}=R_{Z Z}$. To keep things simple, we do not include the experimental efficiencies for the $\gamma \gamma$ channel in Eq. (11), since the efficiencies are different for the two experiments. These are, however, used for the different predictions entering the fit (as described in Eq. (10)). Figure 3 allows to investigate the best fit rates in some more detail (subject to the approximations already discussed). Uncertainty intervals can be extracted from the range with $\Delta \chi_{h}^{2}<1$ (corresponding to $68 \%$ confidence intervals in the Gaussian case). The results for the complete fit are

$$
\begin{array}{ll}
R_{V V}^{h}=0.99_{-0.02}^{+0.22}, & R_{\gamma \gamma}^{h}=1.42_{-0.38}^{+0.12}, \\
R_{b b}^{h}=0.98_{-0.10}^{+0.03}, & R_{\tau \tau}^{h}=0.98_{-0.94}^{+0.01} .
\end{array}
$$

For $R_{\tau \tau}^{h}$ we observe a distribution which is very flat near the minimum. This indicates a low sensitivity in the fit to constraints from $\tau^{+} \tau^{-}$final states, and it permits substantially reduced $\tau^{+} \tau^{-}$rates at a very low additional $\chi^{2}$ contribution. The light Higgs case could therefore easily explain strongly reduced $\tau^{+} \tau^{-}$rates, although this is not visible in the bestfit point.

Results for the heavy Higgs case are shown in Fig. 4. The resulting $\Delta \chi_{H}^{2}$ distributions for individual $R_{X}$ are similar to those for $\Delta \chi_{h}^{2}$, except for $R_{\tau \tau}$, where the additional contribution from the $A$ boson strongly enhances this quantity over the corresponding result in the light Higgs case. Extracting the results for the minimal $\chi^{2}$ in the same way as for the light Higgs case, we obtain for the complete fit

$$
\begin{array}{ll}
R_{V V}^{H}=1.25_{-0.07}^{+0.30}, & R_{\gamma \gamma}^{H}=1.10_{-0.06}^{+0.18}, \\
R_{b b}^{H}=0.83_{-0.12}^{+0.05}, & R_{\tau \tau}^{H / A}=2.54_{-0.17}^{+0.31} .
\end{array}
$$



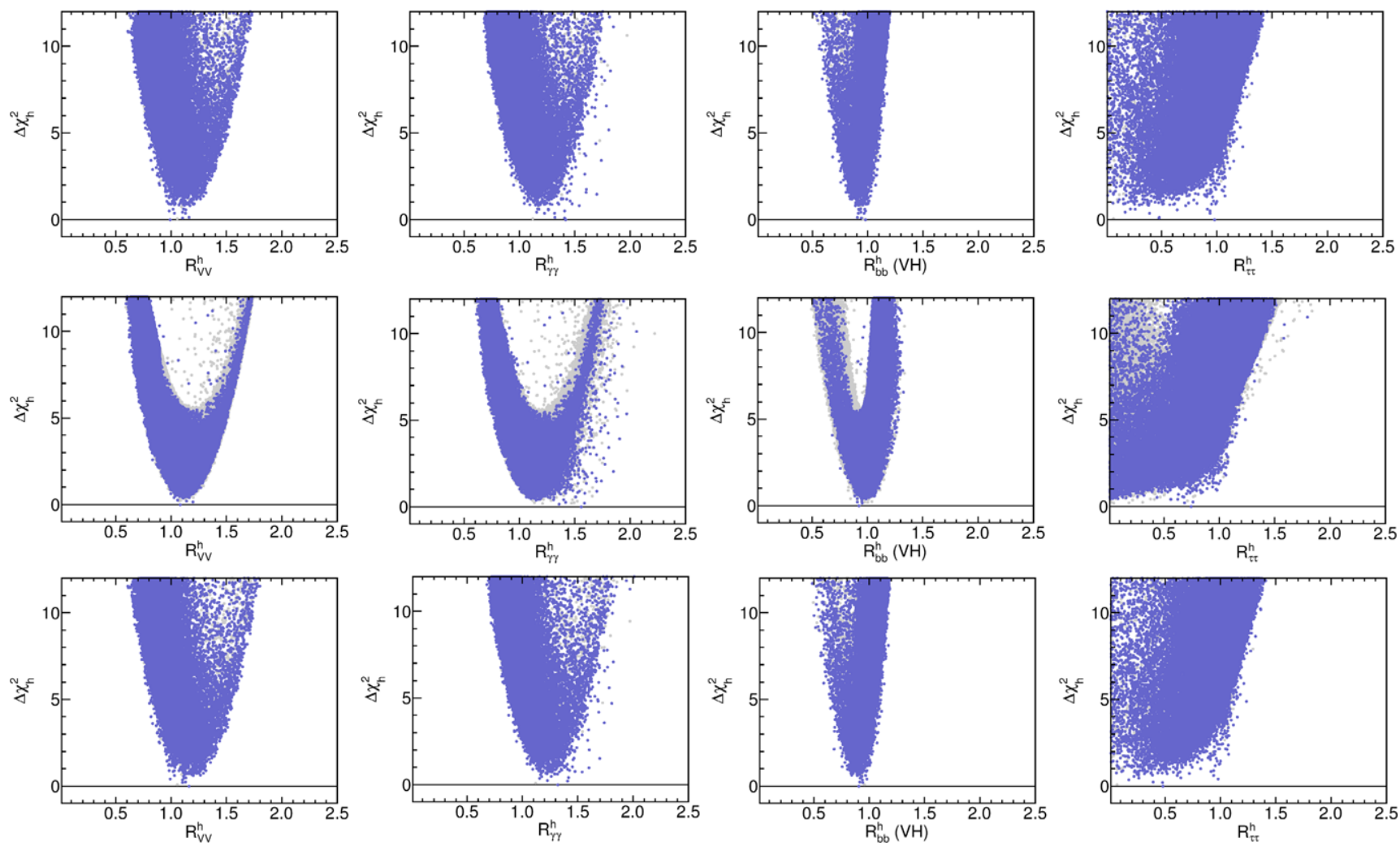

Fig. 3 Distributions of $\Delta \chi_{h}^{2}$ versus the different signal rates (defined in the text) for the light Higgs case. The colours show all points in the scan (grey), and points that pass the direct Higgs search constraints from HiggsBounds (v. 3.8.0) (blue). The three rows show the dis-

An interesting behaviour can be observed in the results leaving out the Tevatron data, as shown in the lower row of Fig. 4. In that case the best fit points have a very small value for $R_{b b}^{H}$. More sampling would be needed to cover this particular case in detail. Since we focus in the following on results of the combined fit, we leave this for a dedicated study.

More information about the phenomenology of the pMSSM-7 Higgs sector can be found from the correlations between the different rates. This is shown in Fig. 5 for the light Higgs case. Compared to the one-dimensional $\chi^{2}$ distributions of Fig. 3, this figure introduces two new colours that are used in the following to show regions close to the minimum $\chi^{2}$. We highlight points for which $\Delta \chi_{h, H}^{2}<2.3$ (red) and $\Delta \chi_{h, H}^{2}<5.99$ (yellow). In the Gaussian limit these correspond to $68 \%$ (95\%) confidence regions with two degrees of freedom. We shall refer to these points simply as the favoured region/points, or sometimes most favoured region/points when $\Delta \chi_{h, H}^{2}<2.3$ is discussed. Here (and in all figures from here on) we refer to the $\chi^{2}$ of the complete fit, including LHC, Tevatron and LEO. The best fit point is indicated in the figures by a black star.

tributions (with the corresponding minimal $\chi^{2}$ subtracted) for the complete fit (upper row), excluding LEO (middle row), and excluding Tevatron data (lower row) (Color figure online)

The left plot of Fig. 5 shows the strong, positive, correlation between $R_{V V}^{h}$ and $R_{\gamma \gamma}^{h}$. In most of the viable parameter space we find $R_{\gamma \gamma}^{h}>R_{V V}^{h}$. The favoured region contains points with fully correlated rates in the interval $0.9 \lesssim R_{\gamma \gamma, V V}^{h} \lesssim 1.6$, but also solutions with lower degree of correlation, where a $\gamma \gamma$ enhancement (up to $R_{\gamma \gamma}^{h} \sim 1.8$ ) is accompanied by a much smaller (or no) enhancement of $R_{V V}^{h}$. In the second plot of Fig. 5 we compare the results of $R_{\gamma \gamma}^{h}$ and $R_{b b}^{h}$ (we remind the reader that the latter rate is calculated using the $V H$ production mode only). We find an anticorrelation between these two rates. This can be understood from the fact that the $h, H \rightarrow b \bar{b}$ decay gives the largest contribution to the total width for a Higgs boson in this mass range, both in the SM and (typically) also in the MSSM. A reduction of the $h, H \rightarrow b \bar{b}$ partial width is therefore effectively a reduction of the total decay width, which leads to a simultaneous enhancement of the branching ratios into the subdominant final states. This has recently been pointed out $[20,48,49]$ as an important mechanism to enhance the $\gamma \gamma$ rate in the MSSM. We shall see below how these effects on the Higgs decay widths affect the parameters in our global fit. The third (right) plot in Fig. 5 shows the weak correlation of $R_{\tau \tau}^{h}$ to $R_{b b}^{h}$, where in principle any 

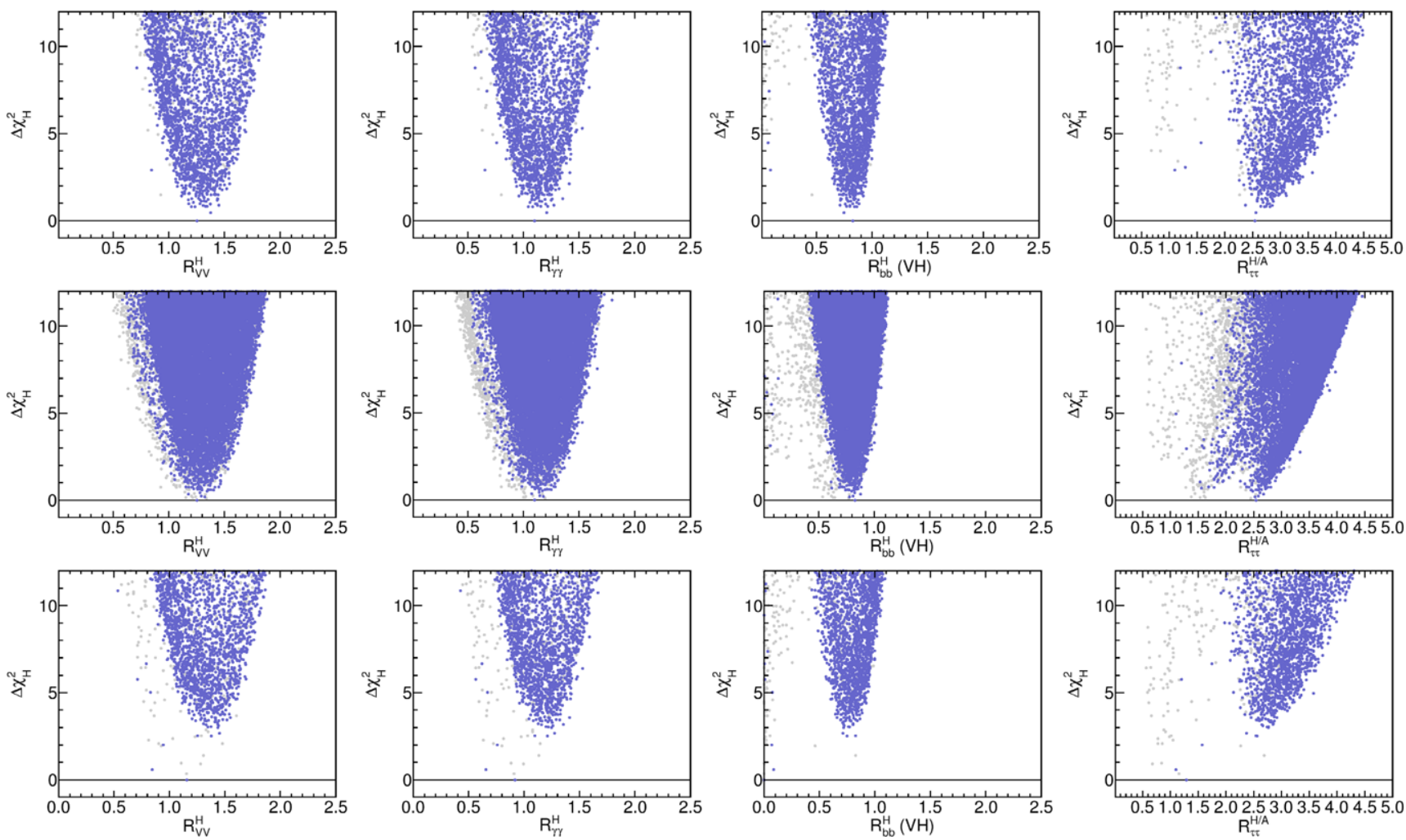

Fig. $4 \Delta \chi_{H}^{2}$ versus the different signal rates (defined in the text) for the heavy Higgs case. Colour coding the same as in Fig. 3. The three rows show the distributions (with the corresponding minimal $\chi^{2}$ sub-

tracted) for the complete fit (upper row), excluding LEO (middle row), and excluding Tevatron data (lower row) (Color figure online)

Fig. 5 Correlations between signal rates for the light Higgs case. The colour coding follows that of Fig. 3, with the addition of the favoured regions with $\Delta \chi_{h}^{2}<2.3$ (red) and

$\Delta \chi_{h}^{2}<5.99$ (yellow). The best fit point is indicated by a black star (Color figure online)
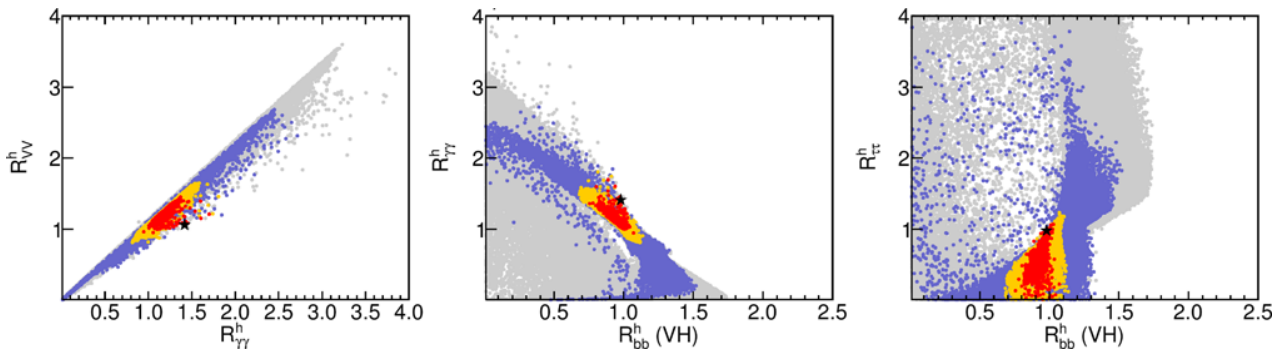

Fig. 6 Correlations between signal rates in the heavy Higgs case. Colours similar to Fig. 5, but here representing $\Delta \chi_{H}^{2}<2.3$ (red) and $\Delta \chi_{H}^{2}<5.99$ (yellow). The black star indicates the best fit point for the heavy Higgs case (Color figure online)
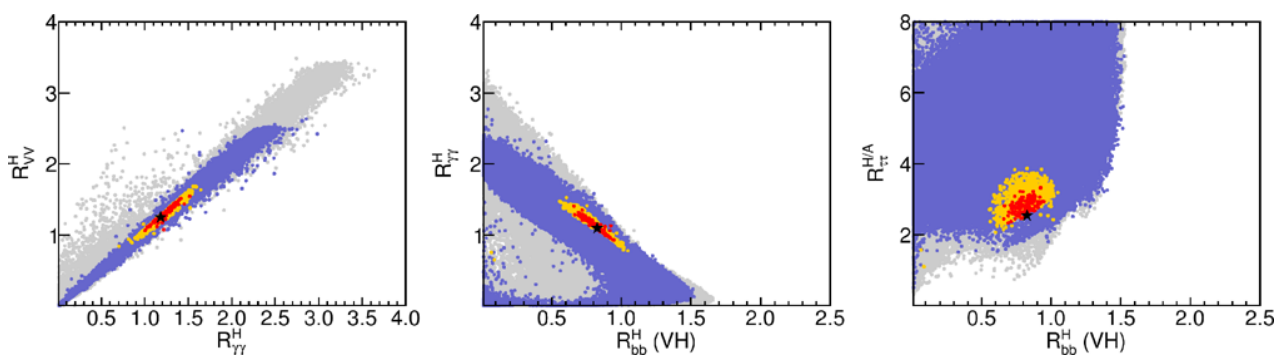

value of $R_{\tau \tau}^{h}<1$ is found in the favoured region for $R_{b b}^{h} \lesssim 1$. Consequently, it is possible to find a strong reduction of the $\tau^{+} \tau^{-}$mode while maintaining a SM-like $b \bar{b}$ rate.

Turning to Fig. 6, we show the rate correlations for the heavy Higgs case. Similar trends as in the light Higgs case are visible in the heavy Higgs data, with the notable difference in the $\tau^{+} \tau^{-}$rate, mainly due to the inclusion of the contribution from the $\mathcal{C P}$-odd Higgs $A$. The favoured regions are found at values for $R_{\tau \tau}^{H / A}$ between 2 and 4 , while $R_{b b}^{H}$ remains below 1 . 
Fig. 7 Effective couplings to vector bosons and gluon pairs of the Higgs boson corresponding to the LHC signal in the light Higgs case (left) and heavy Higgs case (right). The colour coding follows that of Fig. 5

Fig. 8 Higgs sector tree-level parameters $\left(M_{A}, \tan \beta\right)$ in the light Higgs case (left) and in the heavy Higgs case (right)
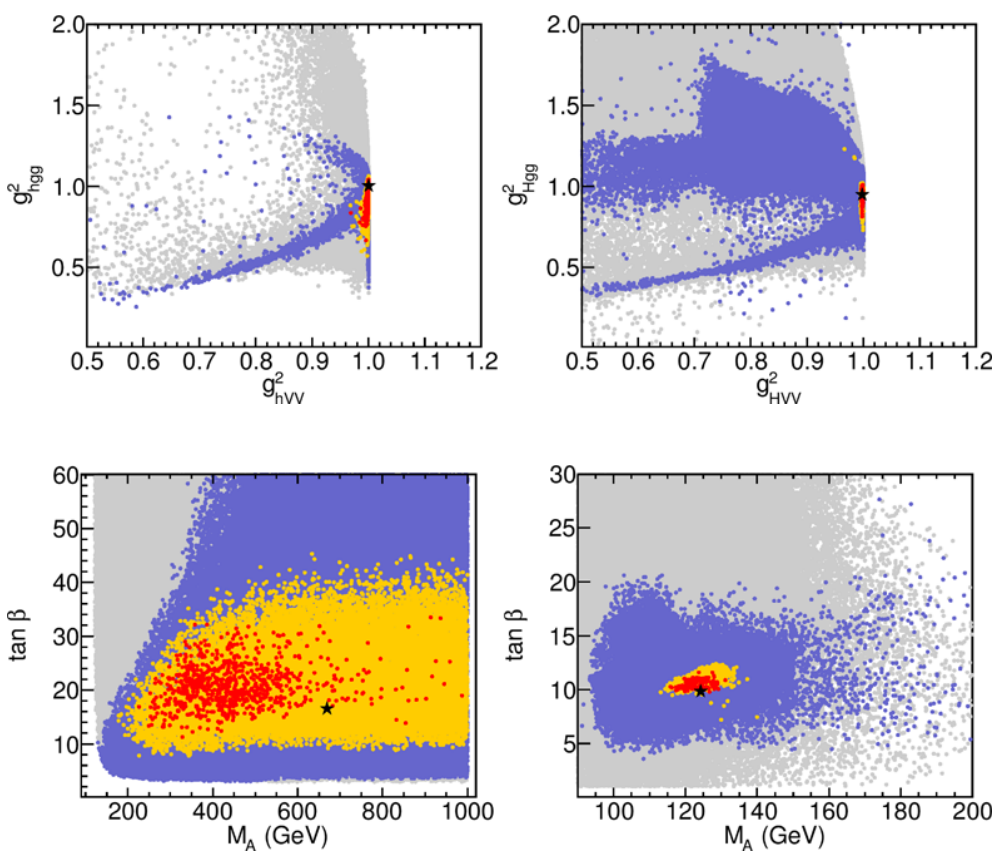

Figure 7 shows the effective couplings to vector bosons and gluons ${ }^{5}$ of the MSSM Higgs assigned to the LHC signal for both the light Higgs case (left plot) and the heavy Higgs case (right plot). In both cases the favoured regions have effective Higgs couplings to vector bosons very close to the SM value $\left(g_{(h, H) V V}^{2}=1\right)$, and the light (heavy) $\mathcal{C P}$ even Higgs boson behaves SM-like in this production (or decay) mode (which reflects the general fact that the $V V h$ or $V V H$ coupling cannot be larger than the corresponding SM coupling). For the effective coupling to gluons, $g_{(h, H) g g}^{2}$, the somewhat larger favoured range $0.7 \lesssim g_{(h, H) g g}^{2} \lesssim 1.1$ is obtained. Consequently, an overall large enhancement of the Higgs production cross sections is disfavoured by the fit. The observed (positive) deviations from the SM in the $V V$ and $\gamma \gamma$ channels for the best fit points can therefore be attributed mainly to changes in the decay branching ratios of the corresponding Higgs states, rather than modifications of the production rates.

We now briefly discuss what mechanisms can alter the branching ratios in the manner observed, and what the consequences are for the favoured regions of MSSM parameter space. In Fig. 8 we show the scan results in the plane of the Higgs sector tree-level parameters $\left(M_{A}, \tan \beta\right)$, where the results for the light (heavy) Higgs case are shown in the left (right) plot. In the light Higgs case one can note the region at low $M_{A}$, high $\tan \beta$, which is excluded by direct MSSM Higgs searches (mainly $H / A \rightarrow \tau^{+} \tau^{-}$). The

\footnotetext{
${ }^{5}$ The effective coupling to gluons is defined via the ratio of the MSSM and SM decay widths to gluons. The relative vector boson coupling is obtained from the inclusion of higher-order corrections into the $\mathcal{C P}$ even Higgs mixing angle, $\alpha$.
}

excluded region appears smaller in this plane than the corresponding results published by the experiments [78, 79], since their results are shown only for one particular benchmark scenario (the so-called $m_{h}^{\max }$ scenario [81]). In an inclusive scan of the pMSSM-7 parameter space, points are found where higher order corrections to the bottom Yukawa coupling lead to suppressed production rates for the heavy MSSM Higgs bosons, and a larger fraction of the parameter space in the $\left(M_{A}, \tan \beta\right)$ plane therefore opens up (see the analyses in $[82,134])$. Sizable branching ratios of $H / A$ to SUSY particles also reduce the sensitivity of the searches in the $\tau^{+} \tau^{-}$final state. We see that the regions of very high $\tan \beta \gtrsim 40$, and also low $\tan \beta \lesssim 8$, are disfavoured by the fit. At high $\tan \beta$ this results from a poor fit to $(g-2)_{\mu}$ and flavour observables, whereas for low $\tan \beta$ the fit to the LHC Higgs observables becomes worse. For low $\tan \beta$ it also becomes increasingly difficult to fit the relatively high Higgs mass value $(125.7 \mathrm{GeV})$, although viable solutions down to $\tan \beta \sim 4$ can be found [19]. Low values of $M_{A}$ are disfavoured by the fit results in the light Higgs case, with the preferred region starting at $M_{A} \gtrsim 170 \mathrm{GeV}$ (and the most favoured region at $M_{A} \gtrsim 230 \mathrm{GeV}$ ). Taking the rate information into account therefore suggests somewhat higher mass scales for the MSSM Higgs sector than what is required by the $\hat{M}_{H} \sim 125.7 \mathrm{GeV}$ Higgs mass measurement alone [19]. For the light Higgs case the lower limits on $M_{A}$ in the favoured regions of the fit exclude the possibility of $M_{H^{ \pm}}<m_{t}$, where the charged Higgs can be produced in the decay of the top quark. On the other hand, the region favoured by the fit does not show any upper limit for $M_{A}$, which demonstrates that the decoupling limit (corresponding to $M_{A} \gg M_{Z}$, where the MSSM Higgs sector re- 


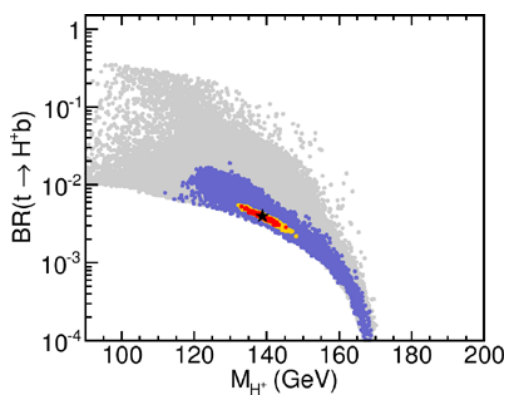

Fig. 9 Branching ratio of the top quark into a charged Higgs boson and a bottom quark in the heavy Higgs case

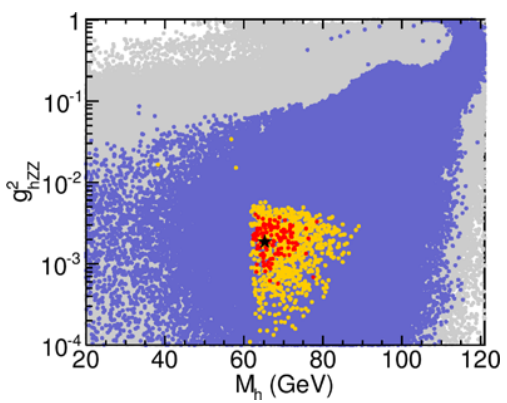

Fig. 10 Effective coupling squared $g_{h Z Z}^{2}$ of the lightest MSSM Higgs boson to a $Z$ boson pair, as a function of the lightest Higgs mass $M_{h}$ in the heavy Higgs case $\left(M_{H} \sim 125.7 \mathrm{GeV}\right)$

produces the predictions for a SM Higgs) remains a possible scenario. This is to be expected, given the high quality of the SM fit to the LHC data.

For the heavy Higgs case, as shown in the right plot of Fig. 8, the situation is very different. Low values for $M_{A}$ are preferred, and the favoured region in $\left(M_{A}, \tan \beta\right)$ is much smaller than for the light Higgs case: $110 \mathrm{GeV} \lesssim M_{A} \lesssim$ $140 \mathrm{GeV}$ and $7 \lesssim \tan \beta \lesssim 13$. Even though the $H$ can be very SM-like in this scenario, this situation is very different from the decoupling limit in the light Higgs case since it implies that all five MSSM Higgs bosons are light. In contrast to the light Higgs case, in this scenario values of the charged Higgs boson mass only below the top mass $\left(M_{H^{ \pm}}<m_{t}\right)$ are found, which may offer good prospects for the searches for charged Higgs bosons in top quark decays. We therefore show in Fig. 9 the results for $\operatorname{BR}\left(t \rightarrow b H^{+}\right)$ as a function of $M_{H^{ \pm}}$. The current upper limit on this decay mode is $\mathcal{O}(1 \%)[135,136]$, which is close to the maximal value favoured by the fit. With more integrated luminosity, charged Higgs searches will therefore have an interesting sensitivity to probe the heavy Higgs scenario at the LHC in the near future.

While in the heavy Higgs scenario the low preferred values for $M_{A}$ typically lead to a situation where $H, A$, and $H^{ \pm}$are rather close in mass, the lightest Higgs boson, $h$, can have a significantly lower mass, as illustrated in Fig. 10. As we see from this figure, points with $M_{h}<90 \mathrm{GeV}$ have a very small effective coupling to vector bosons, $g_{h Z Z}^{2} \ll 1$, which explains why such light Higgs bosons are compatible with the Higgs search limits from LEP. The bulk of the favoured region is found for $60 \mathrm{GeV} \lesssim M_{h} \lesssim 90 \mathrm{GeV}$, with an effective coupling squared to vector bosons at the subpercent level. Another feature which is clearly visible in the HiggsBounds allowed points (blue) is the degradation of the limit around $M_{h} \sim 98 \mathrm{GeV}$, which was caused by a slight excess of events observed at LEP in that mass region. While a scenario with $M_{H} \sim 125.7$ and $M_{h} \sim 98 \mathrm{GeV}$ is certainly possible (see also $[19,51]$ ), it is clearly not favoured by our rate analysis.

The most relevant parameters for higher-order corrections in the MSSM Higgs sector are the soft SUSY-breaking parameters in the stop sector. As it was shown in [19], light scalar top masses down to $150 \mathrm{GeV}$ are in agreement with a light $\mathcal{C P}$-even Higgs mass around $\sim 125 \mathrm{GeV}$, provided the mixing in the scalar top sector is sufficiently strong. This result from the Higgs mass constraint alone (including the exclusion by direct searches) corresponds roughly to the distribution of blue points in Fig. 11. Here we also show the corresponding results including the complete rate analysis. In Fig. 11 we show $X_{t} / M_{\tilde{q}_{3}}$ vs. the light stop mass (left plot) and the light vs. the heavy stop mass (right plot) in the light Higgs case. In the left plot one can see that the case of zero stop mixing in the MSSM is excluded by the observation of a light Higgs at $M_{h} \sim 125.7 \mathrm{GeV}$ (unless $M_{\tilde{q}_{3}}$ is extremely large), and that values of $\left|X_{t} / M_{\tilde{q}_{3}}\right|$ between $\sim 1$ and $\sim 2.5$ must be realised. For the most favoured region we find $X_{t} / M_{\tilde{q}_{3}}=2-2.5$. It should be noted here that large values of $\left|A_{t}\right|\left(\left|A_{t}\right| \gtrsim \sqrt{6} M_{\tilde{q}_{3}}\right)$ could potentially lead to charge and colour breaking minima [137-143]. We checked that applying a cut at $\left|A_{t}\right| \gtrsim \sqrt{6} M_{\tilde{q}_{3}}$ would still leave most points of the favoured region. Concerning the value of the lightest scalar top mass, the overall smallest values are found at $m_{\tilde{t}_{1}} \sim 200 \mathrm{GeV}$, in agreement with [19]. ${ }^{6}$ Even taking the rate information into account, the (most) favoured values start at $m_{\tilde{t}_{1}} \gtrsim 200 \mathrm{GeV}$ for positive $X_{t}$. It is therefore clear that in this case the rate information has a rather limited sensitivity to constrain the minimal stop mass (for positive $X_{t}$ ), and that a good fit is obtained over a large part of the parameter space that is compatible with the allowed range for $M_{h}$.

The right plot in Fig. 11 shows the strong correlation between the two stop masses for the case of degenerate soft mass parameters that we are considering. The light $\tilde{t}_{1}$ is typically accompanied by a somewhat heavier $\tilde{t}_{2}$, but values of $m_{\tilde{t}_{1}} \sim 200 \mathrm{GeV}$ are still realised for $m_{\tilde{t}_{2}} \sim 600 \mathrm{GeV}$, which would mean that both stop masses are rather light, offering interesting possibilities for the LHC. The highest favoured $m_{\tilde{t}_{1}}$ values we find are $\sim 1.4 \mathrm{TeV}$. These are the maximal

\footnotetext{
${ }^{6}$ In this work a Higgs mass measurement of $\hat{M}_{H}=125 \mathrm{GeV}$ was assumed, whereas we now use the average mass $\hat{M}_{H}=125.7 \mathrm{GeV}$.
} 
Fig. 11 Stop mixing parameter $X_{t} / M_{\tilde{q}_{3}}$ vs. the light stop mass (left), and the light vs. heavy stop masses (right) in the light Higgs case
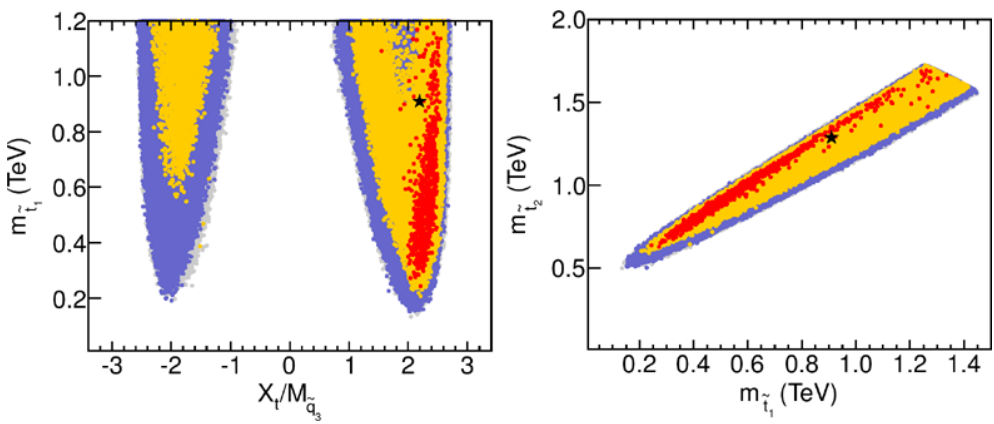

Fig. 12 Stop mixing parameter $X_{t} / M_{\tilde{q}_{3}}$ vs. the light stop mass (left), and the light vs. heavy stop masses (right) in the heavy Higgs case
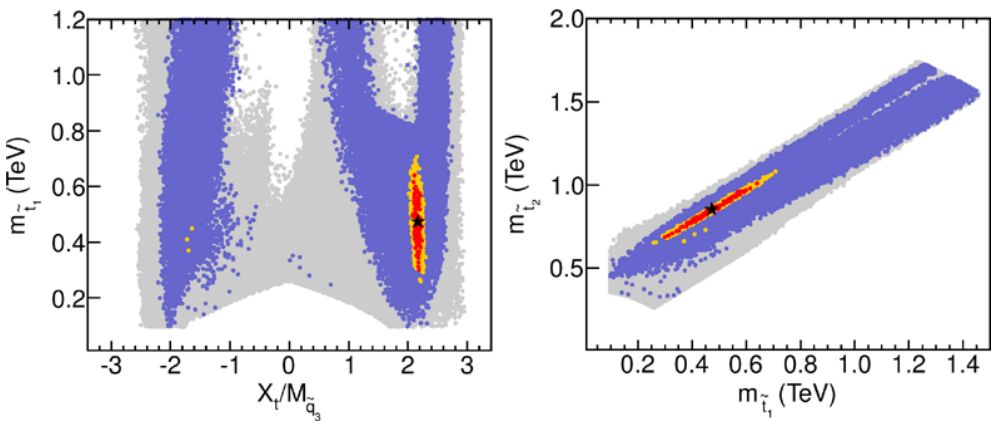

values reached in our scan, but from Fig. 11 it appears plausible that the favoured region extends to larger values of both stop masses. Such a scenario would be extremely difficult to access at the LHC. For the interpretation of these results it is important to remember that we have assumed a universal value for the soft mass parameters in the scalar top and bottom sector. Relaxing this assumption would potentially lead to larger regions of parameter space in which all applied constraints can be satisfied.

The results for the scalar top masses in the heavy Higgs case look in principle similar to the light Higgs case, but with substantially smaller favoured regions, which are nearly solely realised for positive $X_{t}$ with $X_{t} / M_{\tilde{q}_{3}}=2-2.3$, as can be seen in Fig. 12. The favoured values of $m_{\tilde{t}_{1}}$ range between $\sim 250 \mathrm{GeV}$ and $\sim 700 \mathrm{GeV}$ in this case, whereas the preferred range of the heavy stop extends from $m_{\tilde{t}_{2}} \sim 650 \mathrm{GeV}$ to $m_{\tilde{t}_{2}} \sim 1100 \mathrm{GeV}$. Here the difference between the minimal stop mass obtained for the allowed points in the correct Higgs mass range (blue) and in the favoured region is also larger than for the light Higgs case. This shows that, in the heavy Higgs interpretation, the rate observables are more important also for constraining the stop masses.

We now turn to the analysis of rates as a function of the underlying MSSM parameters. This comparison allows to analyse the various mechanisms that are responsible for the observed differences in the decay rates with respect to the SM values. In Fig. 13 we analyse the correlation between the ratio $X_{t} / M_{\tilde{q}_{3}}$ and $R_{\gamma \gamma}^{h}$ (left) or $R_{b b}^{h}(\mathrm{VH})$ (right) in the light Higgs case. It can be seen that the enhancement in the $\gamma \gamma$ channel is only substantial for $X_{t} / M_{\tilde{q}_{3}} \gtrsim 2$, where values of up to $R_{\gamma \gamma}^{h} \sim 1.7$ can be reached in the favoured region.
Such an enhancement can have two sources: a suppression of $\Gamma(h \rightarrow b \bar{b})$, as the by far largest contribution to the total width, or a direct enhancement of $\Gamma(h \rightarrow \gamma \gamma)$. That the first mechanism is indeed responsible for a substantial part of the scenarios with an enhancement of $R_{\gamma \gamma}^{h}$ can be seen in the right plot of Fig. 13, which together with the middle plot of Fig. 5 illustrates that the enhancement in the $\gamma \gamma$ channel in the favoured regions is accompanied by some suppression of the $b \bar{b}$ channel. This suppression/enhancement is realised for large values of $X_{t} / M_{\tilde{q}_{3}}$.

Such a suppression of the $b \bar{b}$ channel can happen in two different ways. In the MSSM, the (effective) coupling $g_{h b \bar{b}}$ is given by

$\frac{g_{h b \bar{b}}}{g_{H_{\mathrm{SM}} b \bar{b}}}=\frac{1}{1+\Delta_{b}}\left(-\frac{\sin \alpha_{\mathrm{eff}}}{\cos \beta}+\Delta_{b} \frac{\cos \alpha_{\mathrm{eff}}}{\sin \beta}\right)$,

where $\alpha$ is the mixing angle in the $\mathcal{C P}$-even Higgs sector. Higher-order contributions from Higgs propagator corrections can approximately be included via the introduction of an effective mixing angle, corresponding to the replacement $\alpha \rightarrow \alpha_{\text {eff [108] (in our numerical analysis we treat }}$ propagator-type corrections of the external Higgs bosons in a more complete way, which is based on wave function normalisation factors that form the $\mathbf{Z}$ matrix [90]). A suppression of the $h \rightarrow b \bar{b}$ channel thus occurs for scenarios with small $\alpha_{\text {eff }}$. Furthermore, genuine corrections to the $h b \bar{b}$ vertex enter Eq. (14) via the quantity $\Delta_{b} \propto \mu \tan \beta$ [144-147], ${ }^{7}$

${ }^{7}$ The dominant contributions to $\Delta_{b}$ beyond one-loop order are the
QCD corrections, given in [148]. Those two-loop contributions are not 
Fig. 13 Dependence of the rates $R_{\gamma \gamma}^{h}$ and $R_{b b}^{h}(\mathrm{VH})$ on the stop mixing parameter $X_{t} / M_{\tilde{q}_{3}}$ for the light Higgs case
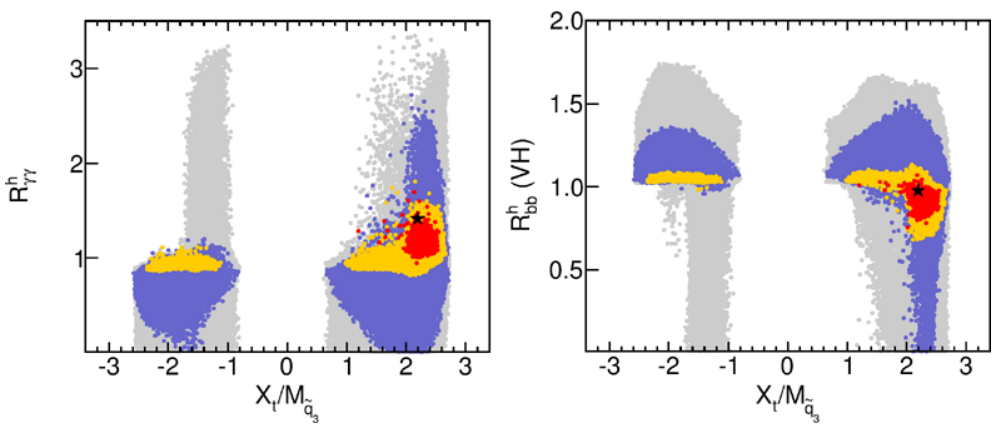

Fig. 14 Correlation of the $\mu$ parameter to the value of $M_{A}$ (left), and dependence of $\Delta_{b}$ corrections on $\mu \tan \beta$ (right), both in the light Higgs case

Fig. 15 Enhancement of the $h \rightarrow \gamma \gamma$ partial width in the presence of light staus for the light Higgs case (left) and heavy Higgs case (right)
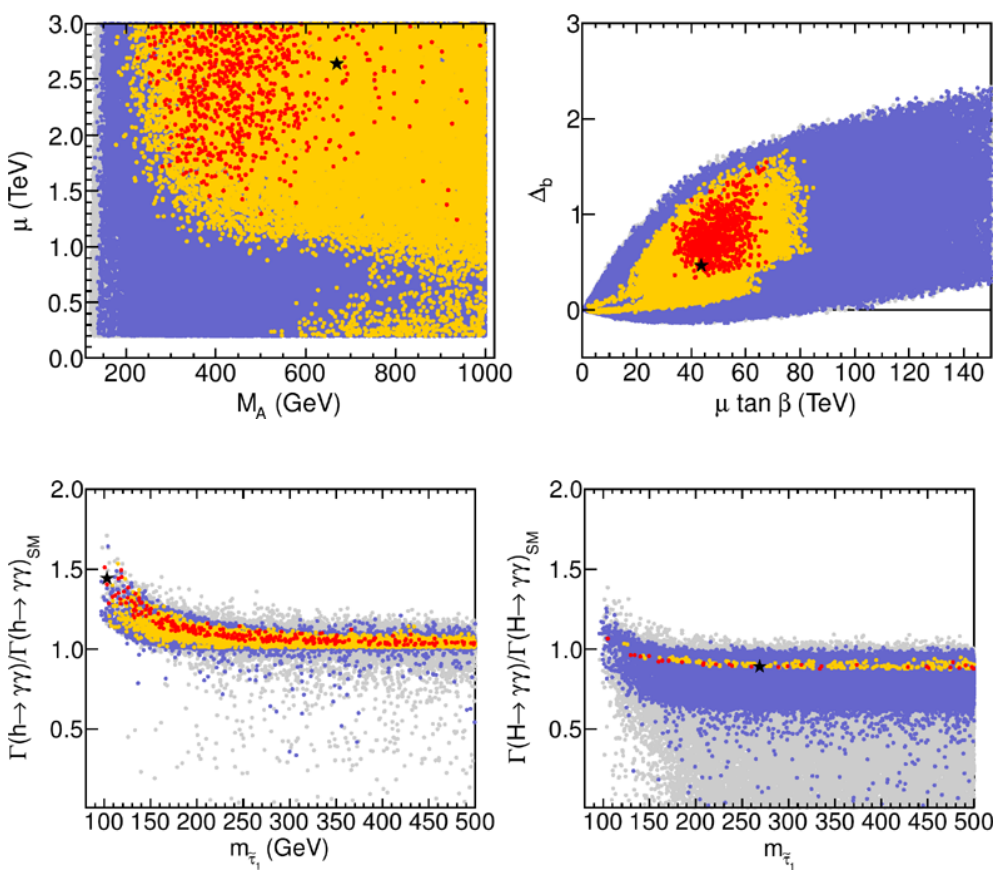

see [20] for a more detailed discussion of the possible mechanisms giving rise to suppression of the $h \rightarrow b \bar{b}$ channel.

While the loop-corrected coupling $g_{h b \bar{b}}$, as given in Eq. (14), approaches the SM coupling in the decoupling limit $\left(M_{A} \gg M_{Z}\right)$, a suppression of $g_{h b \bar{b}}$ is possible for $M_{A}$ not too large provided that $\Delta_{b}$ is numerically sizable and positive. We analyse this in Fig. 14. The left plot in this figure shows that the most favoured regions are obtained for $\mu>1 \mathrm{TeV}$, and that the combination of small $\mu$ and $M_{A}<500 \mathrm{GeV}$ is disfavoured. The corresponding $\Delta_{b}$ values are shown in the right plot as a function of $\mu \tan \beta$. The most favoured regions here are found in the range $0.3 \lesssim \Delta_{b} \lesssim 1.5$, for correspondingly large values of $\mu \tan \beta \sim 30-70 \mathrm{TeV}$. Note that the large values for the $\Delta_{b}$ corrections do not pose problems with perturbativity, since they tend to reduce the bottom Yukawa coupling. It should be noted that the $\Delta_{b}$ corrections in Eq. (14) have another

included in our analysis, but their numerical effect is approximated by using a scale of $m_{t}$ for the evaluation of the one-loop expression. important effect: while in the absence of those contributions a small value of $\alpha_{\text {eff }}$ would give rise to a simultaneous suppression of the Higgs couplings to $b \bar{b}$ and to $\tau^{+} \tau^{-}$, the $\Delta_{b}$ corrections differ from the corresponding contributions to the $g_{h \tau^{+} \tau^{-}}$coupling. This implies in particular that the $g_{h \tau^{+} \tau^{-}}$coupling may be suppressed while the $g_{h b \bar{b}}$ coupling remains unsuppressed (and vice versa), see the discussion of Fig. 5 above.

For the second mechanism, direct enhancement of the $\Gamma(h \rightarrow \gamma \gamma)$ width, it is known that other SUSY particles can play an important role. One possibility that has been discussed recently is to have very light scalar taus $[48,49]$. The effect of light scalar taus can also be observed in our analysis, as can be seen in Fig. 15. Here we show $\Gamma(h, H \rightarrow$ $\gamma \gamma) / \Gamma(h, H \rightarrow \gamma \gamma)_{\mathrm{SM}}$ as a function of $m_{\tilde{\tau}_{1}}$. In the light Higgs case, shown in the left plot, for $m_{\tilde{\tau}_{1}} \sim 100 \mathrm{GeV}$ the enhancement over the SM width reaches $50 \%$ in the favoured region. Even lower values of $m_{\tilde{\tau}_{1}}$ (which are allowed regarding the limits from direct searches, see [86]) are forbidden in our scan from the requirement that the LSP is the lightest neutralino, together with the lower limit of 
Fig. 16 Dependence of the rates $R_{\gamma \gamma}^{H}$ and $R_{b b}^{H}(\mathrm{VH})$ on the stop mixing parameter $X_{t} / M_{\tilde{q}_{3}}$ for the heavy Higgs case

Fig. 17 Correlation of the $\mu$ parameter to the value of $M_{A}$ (left), and dependence of $\Delta_{b}$ corrections on $\mu \tan \beta$ (right), both in the heavy Higgs case
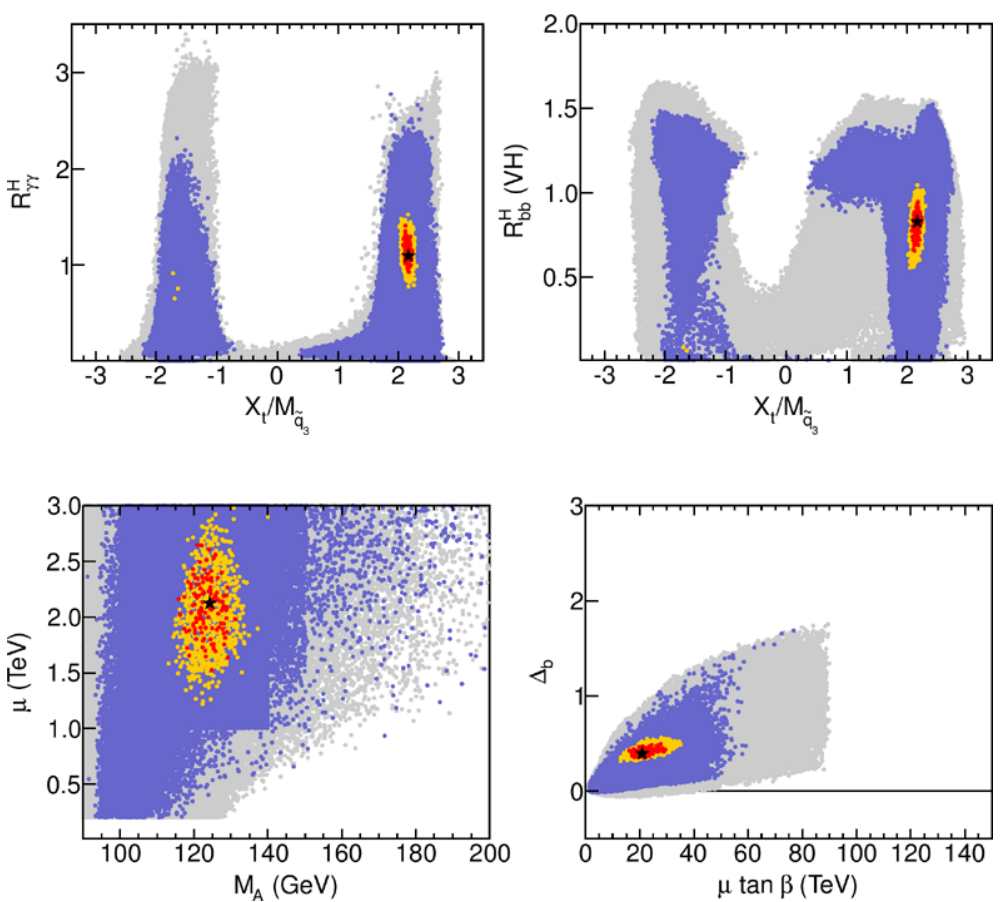

Table 6 Best fit parameter values (in the respective middle column) and ranges for $\Delta \chi_{h, H}^{2}<1$. Values in parentheses indicate that the limit of the scan range has been reached

\begin{tabular}{|c|c|c|c|c|c|c|}
\hline \multirow{3}{*}{$\begin{array}{l}\text { Parameter } \\
M_{A}[\mathrm{GeV}]\end{array}$} & \multicolumn{3}{|c|}{ Light Higgs case } & \multicolumn{3}{|c|}{ Heavy Higgs case } \\
\hline & \multicolumn{3}{|c|}{ Best fit } & \multicolumn{3}{|c|}{ Best fit } \\
\hline & 300 & 669 & 860 & 120.5 & 124.2 & 128.0 \\
\hline $\tan \beta$ & 15 & 16.5 & 26 & 9.7 & 9.8 & 10.8 \\
\hline$\mu[\mathrm{GeV}]$ & 1900 & 2640 & $(3000)$ & 1899 & 2120 & 2350 \\
\hline$M_{\tilde{q}_{3}}[\mathrm{GeV}]$ & 450 & 1100 & (1500) & 580 & 670 & 740 \\
\hline$M_{\tilde{l}_{3}}[\mathrm{GeV}]$ & 250 & 285 & (1500) & (200) & 323 & (1500) \\
\hline$A_{f}[\mathrm{GeV}]$ & 1100 & 2569 & 3600 & 1450 & 1668 & 1840 \\
\hline$M_{2}[\mathrm{GeV}]$ & (200) & 201 & 450 & $(200)$ & 304 & 370 \\
\hline$M_{h}[\mathrm{GeV}]$ & 122.2 & 126.1 & 127.1 & 63.0 & 65.3 & 72.0 \\
\hline$M_{H}[\mathrm{GeV}]$ & 280 & 665 & 860 & 123.9 & 125.8 & 126.4 \\
\hline$M_{H^{ \pm}}[\mathrm{GeV}]$ & 310 & 673 & 860 & 136.5 & 138.8 & 141.5 \\
\hline
\end{tabular}

$M_{2} \geq 200 \mathrm{GeV}$ and the GUT relation between $M_{1}$ and $M_{2}$. Relaxing these assumptions would allow for a larger enhancement of $\Gamma(h \rightarrow \gamma \gamma) / \Gamma(h \rightarrow \gamma \gamma)_{\mathrm{SM}}$, as is clear from the sharp rise of this rate seen in Fig. 15 for low $m_{\tilde{\tau}_{1}}$. For $m_{\tilde{\tau}_{1}} \gtrsim 300 \mathrm{GeV}$ a decoupling to the SM rate is observed. Through the contributions of light scalar taus it is thus possible to accommodate enhanced values of $R_{\gamma \gamma}^{h}$, while maintaining $R_{b b}^{h}$ and $R_{V V}^{h}$ at the SM level (as also observed in our results, cf. Fig. 5). In the heavy Higgs case, on the other hand, as shown in the right plot of Fig. 15, the favoured region is located close to one, and light staus do not contribute to a possible enhancement of $R_{\gamma \gamma}^{H}$.

Similarly to the light Higgs case, we investigate the dependence of the rates on the stop sector parameters for the heavy Higgs case. The results are shown in Fig. 16. As in Fig. 12, the favoured regions are given for large and positive $X_{t} / M_{\tilde{q}_{3}}$, where we find $0.8 \lesssim R_{\gamma \gamma}^{H} \lesssim 1.6$ and a corresponding suppression of $0.6 \lesssim R_{b b}^{H} \lesssim 1.0$. The $\Delta_{b}$ corrections, which enter analogously to Eq. (14), can also in this case be largely responsible for the suppression of the $R_{b \bar{b}}^{H}$ rate, as we show in Fig. 17. Here one can see that in the heavy Higgs scenario only values of $\Delta_{b}$ between $\sim 0.3$ and $\sim 0.6$ are favoured, which are realised for $10 \mathrm{TeV} \lesssim \mu \tan \beta \lesssim 35 \mathrm{TeV}$, i.e. smaller values than in the light Higgs case.

To summarise the discussion on favoured MSSM parameter regions, we list in Table 6 the parameter values for the best fit points (for the complete fit). We also give the parameter ranges corresponding to $\Delta \chi_{h, H}^{2}<1$. For several of the parameters this range extends to the limits of our scanned 
interval. Cases like this have been indicated in Table 6 with parentheses around the corresponding numbers. One can see that in most cases the ranges, even evaluated for $\Delta \chi_{h, H}^{2}<1$, are quite wide. One exception is $\tan \beta$, which is relatively tightly constrained (at least at the level of $\Delta \chi_{h, H}^{2}<1$ ) in the light Higgs case, and even more so in the heavy Higgs case. In the latter case, as discussed above, also the masses of the additional Higgs bosons are relatively tightly constrained, offering some important information on how this scenario can be further explored (see the next section). More precise experimental data would be needed to achieve tighter constraints on the other fitted parameters, which enter the MSSM Higgs phenomenology via loop corrections. The fact that even in the more "exotic" scenario, where the signal at $\sim 125.7 \mathrm{GeV}$ is interpreted in terms of the heavier $\mathcal{C P}$. even Higgs of the MSSM, the values of individual SUSY parameters are only moderately constrained by the fit illustrates that a reasonably good description of the data can be achieved without the need of tuning certain parameters to specific values. This is of course even more the case for the interpretation in terms of the light $\mathcal{C P}$-even Higgs.

\section{Conclusions and outlook}

We have analysed the compatibility of the Minimal Supersymmetric Standard Model (MSSM) with the recent discovery at the LHC of a Higgs-like state at $\hat{M}_{H} \sim 125.7 \mathrm{GeV}$. To this end we have studied the low-energy (phenomenological) pMSSM-7 parameter space, where we allowed the seven parameters most relevant for Higgs and flavour phenomenology to vary freely: the $\mathcal{C P}$-odd Higgs boson mass, $M_{A}$, the ratio of the two vacuum expectation values, $\tan \beta$, a common soft SUSY-breaking parameter for the scalar topand bottom quarks, $M_{\tilde{q}_{3}}$, a soft SUSY-breaking parameter for the scalar tau and neutrino sector, $M_{\tilde{l}_{3}}$, a common trilinear coupling for the third generation, $A_{f}$, the higgsino mass parameter, $\mu$, as well as the $\mathrm{SU}(2)$ gaugino mass parameter, $M_{2}$. The U(1) gaugino mass parameter $M_{1}$ was fixed from the value of $M_{2}$ using the GUT relation. The other parameters have been set to fixed values as to be generically in agreement with recent SUSY searches at the LHC and with low-energy observables such as $(g-2)_{\mu}$.

A random parameter scan over the seven free parameters with $\mathcal{O}\left(10^{7}\right)$ scan points has been performed. For each scan point, a $\chi^{2}$ function was evaluated, taking into account the measured rates in 37 individual Higgs search channels from ATLAS, CMS, and the Tevatron, the best-fit mass values of the LHC experiments, as well as the following low-energy observables: $\mathrm{BR}\left(B \rightarrow X_{s} \gamma\right), \operatorname{BR}\left(B_{s} \rightarrow \mu^{+} \mu^{-}\right), \operatorname{BR}\left(B_{u} \rightarrow\right.$ $\left.\tau v_{\tau}\right),(g-2)_{\mu}$ and $M_{W}$.

As a starting point we find that the SM yields a good fit to the data, with a $\chi^{2}$ per degree of freedom (dof) around unity. The precise value depends on whether low-energy observables and/or the Tevatron data are included in the fit. Turning to the MSSM, we find that the pMSSM-7 provides an excellent fit to the Higgs data in the case that the light $\mathcal{C P}$ even Higgs is interpreted as the new state at $\sim 125.7 \mathrm{GeV}$. In the case that the heavy $\mathcal{C P}$-even Higgs boson is interpreted as the newly discovered state the fit is still acceptable, but somewhat worse than in the light Higgs case once Tevatron and low-energy data are included. The two MSSM best-fit points have a total $\chi^{2} /$ dof of $30.4 / 36(42.4 / 36)$ for the light (heavy) Higgs case, respectively, after the inclusion of LHC, Tevatron and low-energy data. This translates into $p$-values of $73 \%$ and $21 \%$, respectively. The corresponding SM value for $\chi^{2} / v$ is $45.3 / 42$, resulting in a fit probability of $34 \%$. The largest $\chi^{2}$ contribution in the SM comes from the inclusion of $(g-2)_{\mu}$, which shows a more than $3 \sigma$ deviation from the SM prediction. Regarding the comparison of the results for the light Higgs case and the heavy Higgs case in the MSSM it should be noted that a sizable part of the additional $\chi^{2}$ contribution in the heavy Higgs case results from the $\operatorname{BR}\left(B_{u} \rightarrow \tau v_{\tau}\right)$ measurement and from $(g-2)_{\mu}$. The agreement between theory and experiment (both for the MSSM and the SM) for $\mathrm{BR}\left(B_{u} \rightarrow \tau v_{\tau}\right)$ would improve with the inclusion of the new Belle measurement. The $\chi^{2}$ contribution arising from $(g-2)_{\mu}$ for the heavy Higgs case of the MSSM could potentially be improved if in addition to the seven parameters that are varied in our fit also the second generation slepton parameters would be treated as free fit parameters, which would essentially select the slepton mass parameters yielding the lowest $\chi^{2}$ value from $(g-2)_{\mu}$ for each point in parameter space without affecting the other phenomenology. Thus, while the best description of the data is achieved if the new state at $\sim 125.7 \mathrm{GeV}$ is interpreted as the light $\mathcal{C P}$-even Higgs boson of the MSSM, the more "exotic" interpretation in terms of the heavier $\mathcal{C P}$-even Higgs of the MSSM is also permitted by the data, even if the results from the Higgs searches at the LHC are supplemented with results from the Tevatron Higgs searches and with results from flavour physics and electroweak precision data. The latter interpretation would imply that also the other four Higgs bosons of the MSSM would be rather light, giving rise to exciting prospects for the searches for non SM-like Higgses.

In the case of the light $\mathcal{C P}$-even Higgs with $M_{h} \sim$ 125.7 GeV, we find for the best-fit point in the full fit an enhancement of production times branching ratio for the $\gamma \gamma$ channels of about $40 \%$ with respect to the SM prediction. The rates for the gauge boson channels that we obtain are similar to the SM, and the same holds for the fermionic channels $\left(b \bar{b}\right.$ and $\left.\tau^{+} \tau^{-}\right)$. While the fit results for the $\gamma \gamma$, $V V$, and $b \bar{b}$ rates show a clear $\chi^{2}$ minimum, the $\tau^{+} \tau^{-}$ channel has a very broad distribution close to the minimum, and no strong preference can be attributed to the actual bestfit value. 
In the case of the heavy $\mathcal{C P}$-even Higgs with $M_{H} \sim$ 125.7 GeV we find for the best fit point a somewhat smaller enhancement of the $\gamma \gamma$ channel, an enhancement of the gauge boson channels, and a suppression of the $\tau^{+} \tau^{-}$ (VBF) and $b \bar{b}$ channels, whereas the $\tau^{+} \tau^{-}$inclusive channel is enhanced due to the contribution of the $\mathcal{C P}$-odd Higgs boson.

For the light Higgs case, as well as for the heavy Higgs case, the rates in the $\gamma \gamma$ and $V V$ channels are strongly correlated, however, in most cases with the possibility of a stronger enhancement (or smaller suppression) in the $\gamma \gamma$ channel. Between the $\gamma \gamma$ channel and the $b \bar{b}$ channel an anticorrelation can be observed. This shows that the fit within the MSSM favours at least over a part of the preferred region a scenario where a $\gamma \gamma$ enhancement is caused by a suppression of the $b \bar{b}$ channel. Our results furthermore show that the $\tau^{+} \tau^{-}$channel can be strongly suppressed, while the $b \bar{b}$ channel can remain close to the SM strength. Since we find that the $g g \rightarrow h, H$ production channels in the favoured regions are not substantially enhanced, the observed enhancement of the $\gamma \gamma$ channel is not caused by larger production cross sections. A suppression of the $b \bar{b}$ channel in the light and the heavy Higgs case can be caused by a large value of $\Delta_{b}$, which can reach values exceeding unity in the favoured region. In the light Higgs case, the $\gamma \gamma$ channel can also be enhanced by the contribution of light scalar taus to the decay process. In the case where the lightest scalar tau mass is as low as about $100 \mathrm{GeV}$, we find an enhancement of up to $50 \%$ from this mechanism.

For the scalar top masses, we find that the favoured regions start at $m_{\tilde{t}_{1}} \sim 200 \mathrm{GeV}$ and $m_{\tilde{t}_{2}} \sim 600 \mathrm{GeV}$ in the light Higgs case. They extend up to $\sim 1.4 \mathrm{TeV}$ and $\sim 1.6 \mathrm{TeV}$, respectively, which are the maximal values accessible in our scan. The mixing in the scalar top sector must exceed $\left|X_{t} / M_{\tilde{q}_{3}}\right| \sim 1$, where the most favoured regions have $X_{t} / M_{\tilde{q}_{3}}=2-2.5$. Similar values for the lower bounds on the scalar top masses are found in the heavy Higgs case. However, for this case we find that the favoured regions are also bounded from above by (roughly) $m_{\tilde{t}_{2}} \lesssim 1 \mathrm{TeV}$.

As is evident from our analysis (as demonstrated e.g. by Figs. 1 and 2), the fitted rates in the MSSM interpretations are not significantly different from the SM predictions, using the current experimental and theoretical uncertainties. Therefore, if no other new states beyond the current Higgs candidate are discovered in the near future, the question arises how much the precision of the current measurements would need to be improved in order to distinguish the MSSM from the SM, based on precisely measured rates alone. In order to obtain a rough estimate for the answer to this question, we set the hypothetical future central values of the measurements to the MSSM best fit point in the light Higgs case, so that we can investigate the impact of prospective future experimental results in a scenario where this particular MSSM point is actually realised in nature. Then, we scale the full uncertainties of the signal rates measured in the LHC8 (i.e., the channels measured at the LHC with $\sqrt{s}=8 \mathrm{TeV}$ ) channels by a global scale factor and infer the value of the scale factor at which the deviation of the SM prediction from the assumed future measurements with higher precision reaches a significant level. We scale only the LHC8 results (and not LHC7, Tevatron, or LEO) to account for those channels for which an actual improvement of precision is expected in the future (at $\sqrt{s}=8 \mathrm{TeV}$ or higher). To reach $2 \sigma(3 \sigma)$ significance for rejecting the $\mathrm{SM}$, we find that a scale factor of $21 \%(18 \%)$ is required. Reducing the uncertainties to $\sim 20 \%$ of their current values (i.e. a factor five improvement compared to the present situation) could be a long-term goal for the high-luminosity LHC $[149,150]$, at least in some of the important channels. This would already provide a better indication of the preferred model. The goal to achieve the necessary improvement for all channels seems rather challenging, even with an integrated luminosity of $3000 \mathrm{fb}^{-1}[149,150]$ (it should be noted that this would also require significant progress on the theory side to reduce the systematic errors). For the necessary improvement of the statistical, systematical and theoretical precision to go significantly beyond this number, as needed in this case to go towards $5 \sigma$ sensitivity for SM exclusion, would require something close to the expected experimental sensitivity of the International Linear Collider (ILC), see [151] and references therein. The corresponding numbers in the heavy Higgs case are $31 \%$ (26\%), which would be needed to reach a $2 \sigma(3 \sigma)$ rejection of the SM via Higgs rate measurements alone.

While distinguishing the fitted MSSM interpretations from the SM by virtue of improving the precision of the rate measurements alone may be difficult, promising paths to establishing the presence of physics beyond the SM in the Higgs sector are on the one hand the search for additional (non-SM-like) Higgs states and on the other hand the precise measurement of further Higgs properties. Concerning the latter, a promising example would be to measure the Higgs $\mathcal{C P}$ properties in the $\tau^{+} \tau^{-}$final state (assuming that the existence of this decay mode will be confirmed for the observed signal). In the heavy Higgs case, as mentioned in Sect. 3.2, the contributions from $H$ and $A$ add up in channels with poor mass resolution when no $g_{H V V}$ coupling is involved. This is the case for the inclusive production mode with decays into $\tau^{+} \tau^{-}$. There, an analysis of the $\tau$ spin correlations $[152,153]$ (and references therein) could show significant deviations from the SM prediction of $\mathcal{C P}=+1$, due to the presence of the $A$ production mode. In contrast, in the light Higgs case, the discovered state at $\hat{M}_{H} \sim 125.7 \mathrm{GeV}$ would be measured with exact $\mathcal{C P}=+1$.

The potential discovery of additional Higgs like states would be the clearest way to distinguish the $\operatorname{MSSM} h$ and $H$ 
interpretations from each other. As discussed above, see in particular Table 6, the masses of the yet undiscovered Higgs bosons are expected in different ranges for the $h$ and $H$ interpretations. However, discovering the lighter $\mathcal{C P}$-even state $h$ in the heavy Higgs case appears to be difficult at the LHC due to its low mass and heavily suppressed coupling to vector bosons, $g_{h V V}^{2}$. At the ILC, however, the $h A$ and $\mathrm{H}^{+} \mathrm{H}^{-}$production modes would be straightforward to measure in the heavy Higgs case. On the other hand, in the light Higgs case all other states could be beyond the mass reach within the current fit uncertainties. Here, the ILC measurements would have to rely on precise coupling measurements to distinguish the $h$ and $H$ interpretations directly (see [151] and references therein). In that case also a combined interpretation of LHC and ILC data might be very valuable [154, 155].

In the heavy Higgs interpretation, the LHC searches for MSSM Higgs bosons in the $\tau^{+} \tau^{-}$final states will soon have sensitivity to start probing the region of small $M_{A}$ and moderate $\tan \beta$, which is favoured in this scenario. Furthermore we find that the charged Higgs boson in this case should be lighter than $m_{t}$, so that at the production from top quark decays at the LHC would be kinematically possible. The favoured values of $\mathrm{BR}\left(t \rightarrow H^{+} b\right)$ are just below the current experimental bounds. Still, an improvement of the limits by one order of magnitude would be required to fully cover this possibility.

New data from the ATLAS and CMS Higgs boson searches is rapidly emerging. It will be particularly important to investigate on the one hand potential deviations of the rates from the SM predictions and on the other hand the outcome of searches for additional non-SM-like Higgses. Confronting these results with predictions in the MSSM will show whether this model, whose unambigous prediction of a light (and potentially SM-like) Higgs boson seems to be well supported by the data, will continue to provide a viable description of nature also in the future.

Acknowledgements This work has been supported by the Collaborative Research Center SFB676 of the DFG, "Particles, Strings, and the Early Universe". It has also been partially funded by the Helmholtz Alliance "Physics at the Terascale". The work of S.H. was partially supported by CICYT (grant FPA 2010-22163-C02-01) and by the Spanish MICINN's Consolider-Ingenio 2010 Programme under grant MultiDark CSD2009-00064. The research of O.S. is supported by the Swedish Research Council (VR) through the Oskar Klein Centre.

\section{References}

1. G. Aad et al. (ATLAS Collaboration), Phys. Lett. B 716, 1-29 (2012). arXiv:1207.7214

2. S. Chatrchyan et al. (CMS Collaboration), Phys. Lett. B 716, 3061 (2012). arXiv: 1207.7235

3. TEVNPH Working Group for the CDF, DØ Collaborations, arXiv:1207.0449
4. ATLAS Collaboration, ATLAS-CONF-2012-091

5. CMS Collaboration, CMS-HIG-12-015

6. ATLAS Collaboration, ATLAS-CONF-2012-093

7. CMS Collaboration, CMS-HIG-12-020

8. J.R. Ellis, G. Ridolfi, F. Zwirner, Phys. Lett. B 257, 83-91 (1991)

9. Y. Okada, M. Yamaguchi, T. Yanagida, Prog. Theor. Phys. 85, $1-6(1991)$

10. H.E. Haber, R. Hempfling, Phys. Rev. Lett. 66, 1815-1818 (1991)

11. A. Brignole, Phys. Lett. B 281, 284-294 (1992)

12. P.H. Chankowski, S. Pokorski, J. Rosiek, Phys. Lett. B 286, 307314 (1992)

13. P.H. Chankowski, S. Pokorski, J. Rosiek, Nucl. Phys. B 423, 437-496 (1994). hep-ph/9303309

14. A. Dabelstein, Z. Phys. C 67, 495-512 (1995). hep-ph/9409375

15. A. Dabelstein, Nucl. Phys. B 456, 25-56 (1995). hep-ph/ 9503443

16. A. Djouadi, Phys. Rep. 459, 1-241 (2008). hep-ph/0503173

17. S. Heinemeyer, Int. J. Mod. Phys. A 21, 2659-2772 (2006). hep-ph/0407244

18. S. Heinemeyer, W. Hollik, G. Weiglein, Phys. Rep. 425, 265-368 (2006). hep-ph/0412214

19. S. Heinemeyer, O. Stål, G. Weiglein, Phys. Lett. B 710, 201-206 (2012). arXiv: 1112.3026

20. R. Benbrik, M. Gomez Bock, S. Heinemeyer, O. Stål, G. Weiglein, L. Zeune, Eur. Phys. J. C 72, 2171 (2012). arXiv: 1207.1096

21. L.J. Hall, D. Pinner, J.T. Ruderman, J. High Energy Phys. 1204, 131 (2012). arXiv: 1112.2703

22. H. Baer, V. Barger, A. Mustafayev, Phys. Rev. D 85, 075010 (2012). arXiv:1112.3017

23. A. Arbey, M. Battaglia, A. Djouadi, F. Mahmoudi, J. Quevillon, Phys. Lett. B 708, 162-169 (2012). arXiv:1112.3028

24. P. Draper, P. Meade, M. Reece, D. Shih, Phys. Rev. D 85, 095007 (2012). arXiv:1112.3068

25. M. Kadastik, K. Kannike, A. Racioppi, M. Raidal, J. High Energy Phys. 1205, 061 (2012). arXiv:1112.3647

26. J. Cao, Z. Heng, D. Li, J.M. Yang, Phys. Lett. B 710, 665-670 (2012). arXiv:1112.4391

27. F. Brümmer, W. Buchmüller, J. High Energy Phys. 1205, 006 (2012). arXiv:1201.4338

28. J. Ellis, K.A. Olive, Eur. Phys. J. C 72, 2005 (2012). arXiv: 1202.3262

29. N. Desai, B. Mukhopadhyaya, S. Niyogi, arXiv: 1202.5190

30. T. Cheng, J. Li, T. Li, D.V. Nanopoulos, C. Tong, arXiv: 1202.6088

31. M. Asano, S. Matsumoto, M. Senami, H. Sugiyama, Phys. Rev. D 86, 015020 (2012). arXiv:1202.6318

32. J.-J. Cao, Z. Heng, J.M. Yang, J. Zhu, J. High Energy Phys. 1206, 145 (2012). arXiv: 1203.0694

33. A. Choudhury, A. Datta, J. High Energy Phys. 1206, 006 (2012). arXiv: 1203.4106

34. M.A. Ajaib, I. Gogoladze, F. Nasir, Q. Shafi, Phys. Lett. B 713, 462-468 (2012). arXiv:1204.2856

35. F. Brümmer, S. Kraml, S. Kulkarni, J. High Energy Phys. 1208, 089 (2012). arXiv:1204.5977

36. J.L. Evans, M. Ibe, T.T. Yanagida, Phys. Rev. D 86, 015017 (2012). arXiv:1204.6085

37. A. Fowlie, M. Kazana, K. Kowalska, S. Munir, L. Roszkowski et al., arXiv:1206.0264

38. M.R. Buckley, D. Hooper, arXiv:1207.1445

39. S. Akula, P. Nath, G. Peim, Phys. Lett. B 717, 188-192 (2012). arXiv:1207.1839

40. J. Cao, Z. Heng, J.M. Yang, J. Zhu, J. High Energy Phys. 1210, 079 (2012). arXiv: 1207.3698

41. M. Hirsch, F. Joaquim, A. Vicente, arXiv:1207.6635 
42. O. Buchmueller, R. Cavanaugh, M. Citron, A. De Roeck, M. Dolan et al., arXiv:1207.7315

43. K. Howe, P. Saraswat, J. High Energy Phys. 1210, 065 (2012). arXiv: 1208.1542

44. C. Wymant, arXiv:1208.1737

45. Z. Kang, T. Li, J. Li, Y. Liu, arXiv: 1208.2673

46. T. Kitahara, arXiv: 1208.4792

47. Z. Heng, arXiv: 1210.3751

48. M. Carena, S. Gori, N.R. Shah, C.E. Wagner, L.-T. Wang, J. High Energy Phys. 1207, 175 (2012). arXiv: 1205.5842

49. M. Carena, S. Gori, N.R. Shah, C.E. Wagner, J. High Energy Phys. 1203, 014 (2012). arXiv: 1112.3336

50. U. Haisch, F. Mahmoudi, arXiv: 1210.7806

51. M. Drees, arXiv: 1210.6507

52. J. Espinosa, C. Grojean, M. Mühlleitner, M. Trott, J. High Energy Phys. 1212, 045 (2012). arXiv:1207.1717

53. D. Carmi, A. Falkowski, E. Kuflik, T. Volansky, J. High Energy Phys. 1207, 136 (2012). arXiv:1202.3144

54. A. Azatov, R. Contino, J. Galloway, J. High Energy Phys. 1204, 127 (2012). arXiv: 1202.3415

55. P.P. Giardino, K. Kannike, M. Raidal, A. Strumia, J. High Energy Phys. 1206, 117 (2012). arXiv: 1203.4254

56. M. Klute, R. Lafaye, T. Plehn, M. Rauch, D. Zerwas, Phys. Rev. Lett. 109, 101801 (2012). arXiv: 1205.2699

57. A. Azatov, S. Chang, N. Craig, J. Galloway, Phys. Rev. D 86, 075033 (2012). arXiv: 1206.1058

58. D. Carmi, A. Falkowski, E. Kuflik, T. Volansky, arXiv: 1206.4201

59. I. Low, J. Lykken, G. Shaughnessy, Phys. Rev. D 86, 093012 (2012). arXiv:1207.1093

60. T. Corbett, O. Eboli, J. Gonzalez-Fraile, M. Gonzalez-Garcia, Phys. Rev. D 86, 075013 (2012). arXiv:1207.1344

61. P.P. Giardino, K. Kannike, M. Raidal, A. Strumia, Phys. Lett. B 718, 469-474 (2012). arXiv: 1207.1347

62. J. Ellis, T. You, J. High Energy Phys. 1209, 123 (2012). arXiv: 1207.1693

63. M. Montull, F. Riva, J. High Energy Phys. 1211, 018 (2012). arXiv: 1207.1716

64. D. Carmi, A. Falkowski, E. Kuflik, T. Volansky, J. Zupan, J. High Energy Phys. 1210, 196 (2012). arXiv:1207.1718

65. S. Banerjee, S. Mukhopadhyay, B. Mukhopadhyaya, J. High Energy Phys. 1210, 062 (2012). arXiv:1207.3588

66. F. Bonnet, T. Ota, M. Rauch, W. Winter, Phys. Rev. D 86, 093014 (2012). arXiv: 1207.4599

67. T. Plehn, M. Rauch, Europhys. Lett. 100, 11002 (2012). arXiv: 1207.6108

68. J.R. Espinosa, C. Grojean, V. Sanz, M. Trott, J. High Energy Phys. 1212, 077 (2012). arXiv: 1207.7355

69. A. Djouadi, arXiv: 1208.3436

70. W. Altmannshofer, S. Gori, G.D. Kribs, Phys. Rev. D 86, 115009 (2012). arXiv: 1210.2465

71. B.A. Dobrescu, J.D. Lykken, arXiv: 1210.3342

72. S. Chang, S.K. Kang, J.-P. Lee, K.Y. Lee, S.C. Park et al., arXiv: 1210.3439

73. G. Cacciapaglia, A. Deandrea, G.D. La Rochelle, J.-B. Flament, arXiv: 1210.8120

74. A. David et al. (LHC Higgs Cross Section Working Group), arXiv: 1209.0040

75. S. Schael et al. (ALEPH Collaboration, DELPHI Collaboration, L3 Collaboration OPAL Collaborations), Eur. Phys. J. C 47, $547-$ 587 (2006). hep-ex/0602042

76. D. Benjamin et al. (TEVNPH Working Group), arXiv:1003.3363

77. T. Aaltonen, et al. (CDF and D0 Collaborations), arXiv: 1207.2757

78. ATLAS Collaboration ATLAS-CONF-2012-094

79. CMS Collaboration CMS-PAS-HIG-11-029
80. M.S. Carena, S. Heinemeyer, C.E.M. Wagner, G. Weiglein, hep-ph/9912223

81. M.S. Carena, S. Heinemeyer, C.E.M. Wagner, G. Weiglein, Eur. Phys. J. C 26, 601-607 (2003). hep-ph/0202167

82. M.S. Carena, S. Heinemeyer, C. Wagner, G. Weiglein, Eur. Phys. J. C 45, 797-814 (2006). hep-ph/0511023

83. A. Arbey, M. Battaglia, F. Mahmoudi, Eur. Phys. J. C 72, 2169 (2012). arXiv:1205.2557

84. A. Arbey, M. Battaglia, A. Djouadi, F. Mahmoudi, J. High Energy Phys. 1209, 107 (2012). arXiv: 1207.1348

85. S. AbdusSalam, B. Allanach, H. Dreiner, J. Ellis, U. Ellwanger et al., Eur. Phys. J. C 71, 1835 (2011). arXiv:1109.3859

86. J. Beringer et al. (Particle Data Group), Phys. Rev. D 86, 010001 (2012)

87. S. Heinemeyer, W. Hollik, G. Weiglein, Eur. Phys. J. C 9, $343-$ 366 (1999). hep-ph/9812472

88. S. Heinemeyer, W. Hollik, G. Weiglein, Comput. Phys. Commun. 124, 76-89 (2000). hep-ph/9812320

89. G. Degrassi, S. Heinemeyer, W. Hollik, P. Slavich, G. Weiglein, Eur. Phys. J. C 28, 133-143 (2003). hep-ph/0212020

90. M. Frank, T. Hahn, S. Heinemeyer, W. Hollik, H. Rzehak et al., J. High Energy Phys. 0702, 047 (2007). hep-ph/0611326

91. P. Bechtle, O. Brein, S. Heinemeyer, G. Weiglein, K.E. Williams, Comput. Phys. Commun. 181, 138-167 (2010). arXiv:0811.4169

92. P. Bechtle, O. Brein, S. Heinemeyer, G. Weiglein, K.E. Williams, Comput. Phys. Commun. 182, 2605-2631 (2011). arXiv: 1102.1898

93. ATLAS Collaboration, ATLAS-CONF-2012-012

94. ATLAS Collaboration, ATLAS-CONF-2012-098

95. G. Aad et al. (ATLAS Collaboration), Phys. Rev. D 86, 032003 (2012). arXiv:1207.0319

96. ATLAS Collaboration ATLAS-CONF-2012-092

97. S. Dittmaier et al. (LHC Higgs Cross Section Working Group), arXiv: 1101.0593

98. S. Dittmaier, S. Dittmaier, C. Mariotti, G. Passarino, R. Tanaka et al., arXiv:1201.3084

99. https://twiki.cern.ch/twiki/bin/view/LHCPhysics/CrossSections

100. D. de Florian, M. Grazzini, Phys. Lett. B 674, 291-294 (2009). arXiv:0901.2427

101. http://theory.fi.infn.it/grazzini/hcalculators.html

102. T. Hahn, S. Heinemeyer, F. Maltoni, G. Weiglein, S. Willenbrock, hep-ph/0607308

103. T. Hahn, S. Heinemeyer, W. Hollik, H. Rzehak, G. Weiglein, Nucl. Phys. B, Proc. Suppl. 205-206, 152-157 (2010). arXiv: 1007.0956

104. R. Bonciani, G. Degrassi, A. Vicini, J. High Energy Phys. 0711, 095 (2007). arXiv:0709.4227

105. U. Aglietti, R. Bonciani, G. Degrassi, A. Vicini, J. High Energy Phys. 0701, 021 (2007). hep-ph/0611266

106. A. Dedes, P. Slavich, Nucl. Phys. B 657, 333-354 (2003). hep-ph/0212132

107. A. Dedes, G. Degrassi, P. Slavich, Nucl. Phys. B 672, 144-162 (2003). hep-ph/0305127

108. S. Heinemeyer, W. Hollik, G. Weiglein, Eur. Phys. J. C 16, 139153 (2000). hep-ph/0003022

109. K.E. Williams, H. Rzehak, G. Weiglein, Eur. Phys. J. C 71, 1669 (2011). arXiv:1103.1335

110. Y. Yook, Talk given at ICHEP 2012, see: http://indico.cern.ch/ getFile.py/access? contribId $=573 \&$ sessionId $=66 \&$ resId $=0 \&$ materialId=slides\&confId $=181298$

111. F. Mahmoudi, Comput. Phys. Commun. 178, 745-754 (2008). arXiv:0710.2067

112. F. Mahmoudi, Comput. Phys. Commun. 180, 1579-1613 (2009). arXiv:0808.3144

113. F. Mahmoudi, Comput. Phys. Commun. 180, 1718-1719 (2009) 
114. M. Misiak, M. Steinhauser, Nucl. Phys. B 764, 62-82 (2007). hep-ph/0609241

115. M. Benayoun, P. David, L. DelBuono, F. Jegerlehner, arXiv: 1210.7184

116. G. Degrassi, G. Giudice, Phys. Rev. D 58, 053007 (1998). hep-ph/9803384

117. S. Heinemeyer, D. Stöckinger, G. Weiglein, Nucl. Phys. B 690, 62-80 (2004). hep-ph/0312264

118. S. Heinemeyer, D. Stöckinger, G. Weiglein, Nucl. Phys. B 699, 103-123 (2004). hep-ph/0405255

119. D. Stöckinger, J. Phys. G 34, R45-R92 (2007). hep-ph/0609168

120. Tevatron Electroweak Working Group for the CDF Collaboration, DØ Collaboration, arXiv: 1204.0042

121. http://lepewwg.web.cern.ch/LEPEWWG/

122. M. Awramik, M. Czakon, A. Freitas, G. Weiglein, Phys. Rev. D 69, 053006 (2004). hep-ph/0311148

123. A. Djouadi, P. Gambino, S. Heinemeyer, W. Hollik, C. Junger et al., Phys. Rev. Lett. 78, 3626-3629 (1997). hep-ph/9612363

124. A. Djouadi, P. Gambino, S. Heinemeyer, W. Hollik, C. Junger et al., Phys. Rev. D 57, 4179-4196 (1998). hep-ph/9710438

125. S. Heinemeyer, W. Hollik, D. Stöckinger, A. Weber, G. Weiglein, J. High Energy Phys. 0608, 052 (2006). hep-ph/0604147

126. G. Weiglein, L. Zeune, In preparation

127. Y. Amhis et al. (Heavy Flavor Averaging Group), arXiv: 1207.1158

128. LHCb Collaboration, LHCb-CONF-2012-017

129. CMS Collaboration, CMS-PAS-BPH-12-009

130. ATLAS Collaboration, ATLAS-CONF-2012-061

131. M. Davier, A. Hoecker, B. Malaescu, Z. Zhang, Eur. Phys. J. C 71, 1515 (2011). arXiv: 1010.4180

132. G. Bennett et al. (Muon $g-2$ Collaboration), Phys. Rev. Lett. 92, 161802 (2004). hep-ex/0401008

133. G. Bennett et al. (Muon $g-2$ Collaboration), Phys. Rev. D 73, 072003 (2006). hep-ex/0602035

134. S. Gennai, S. Heinemeyer, A. Kalinowski, R. Kinnunen, S. Lehti et al., Eur. Phys. J. C 52, 383-395 (2007). arXiv:0704.0619

135. G. Aad et al. (ATLAS Collaboration), J. High Energy Phys. 1206, 039 (2012). arXiv:1204.2760
136. S. Chatrchyan et al. (CMS Collaboration), J. High Energy Phys. 1207, 143 (2012). arXiv:1205.5736

137. J. Frere, D. Jones, S. Raby, Nucl. Phys. B 222, 11 (1983)

138. M. Claudson, L.J. Hall, I. Hinchliffe, Nucl. Phys. B 228, 501 (1983)

139. C. Kounnas, A. Lahanas, D.V. Nanopoulos, M. Quiros, Nucl. Phys. B 236, 438 (1984)

140. J. Gunion, H. Haber, M. Sher, Nucl. Phys. B 306, 1 (1988)

141. J. Casas, A. Lleyda, C. Munoz, Nucl. Phys. B 471, 3-58 (1996). hep-ph/9507294

142. P. Langacker, N. Polonsky, Phys. Rev. D 50, 2199-2217 (1994). hep-ph/9403306

143. A. Strumia, Nucl. Phys. B 482, 24-38 (1996). hep-ph/9604417

144. R. Hempfling, Phys. Rev. D 49, 6168-6172 (1994)

145. L.J. Hall, R. Rattazzi, U. Sarid, Phys. Rev. D 50, 7048-7065 (1994). hep-ph/9306309

146. M.S. Carena, M. Olechowski, S. Pokorski, C. Wagner, Nucl. Phys. B 426, 269-300 (1994). hep-ph/9402253

147. M.S. Carena, D. Garcia, U. Nierste, C.E.M. Wagner, Nucl. Phys. B 577, 88-120 (2000). hep-ph/9912516

148. D. Noth, M. Spira, Phys. Rev. Lett. 101, 181801 (2008). arXiv:0808.0087

149. ATLAS Collaboration, Contribution to the ESPP2012, ATLPHYS-PUB-2012-004, http://indico.cern.ch/contributionDisplay. py? contribId $=174 \&$ confId $=175067$

150. CMS Collaboration, Contribution to the ESPP2012, http://indico. cern.ch/abstractDisplay.py/getAttachedFile?abstractId=144\& resId $=1 \& \operatorname{confId}=175067$

151. J.E. Brau, R.M. Godbole, F.R.L. Diberder, M. Thomson, H. Weerts et al., arXiv: 1210.0202

152. S. Berge, W. Bernreuther, J. Ziethe, Phys. Rev. Lett. 100, 171605 (2008). arXiv:0801.2297

153. S. Berge, W. Bernreuther, B. Niepelt, H. Spiesberger, Phys. Rev. D 84, 116003 (2011). arXiv:1108.0670

154. G. Weiglein et al. (LHC/LC Study Group), Phys. Rep. 426, 47358 (2006). hep-ph/0410364

155. K. Desch, E. Gross, S. Heinemeyer, G. Weiglein, L. Zivkovic, J. High Energy Phys. 0409, 062 (2004). hep-ph/0406322 